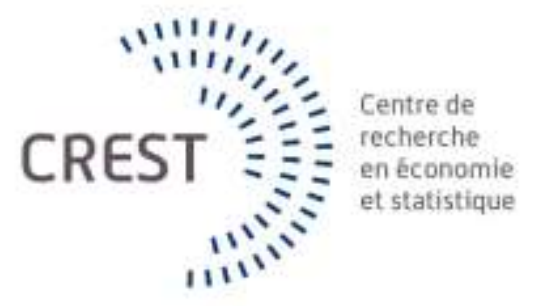

Série des Documents de Travail

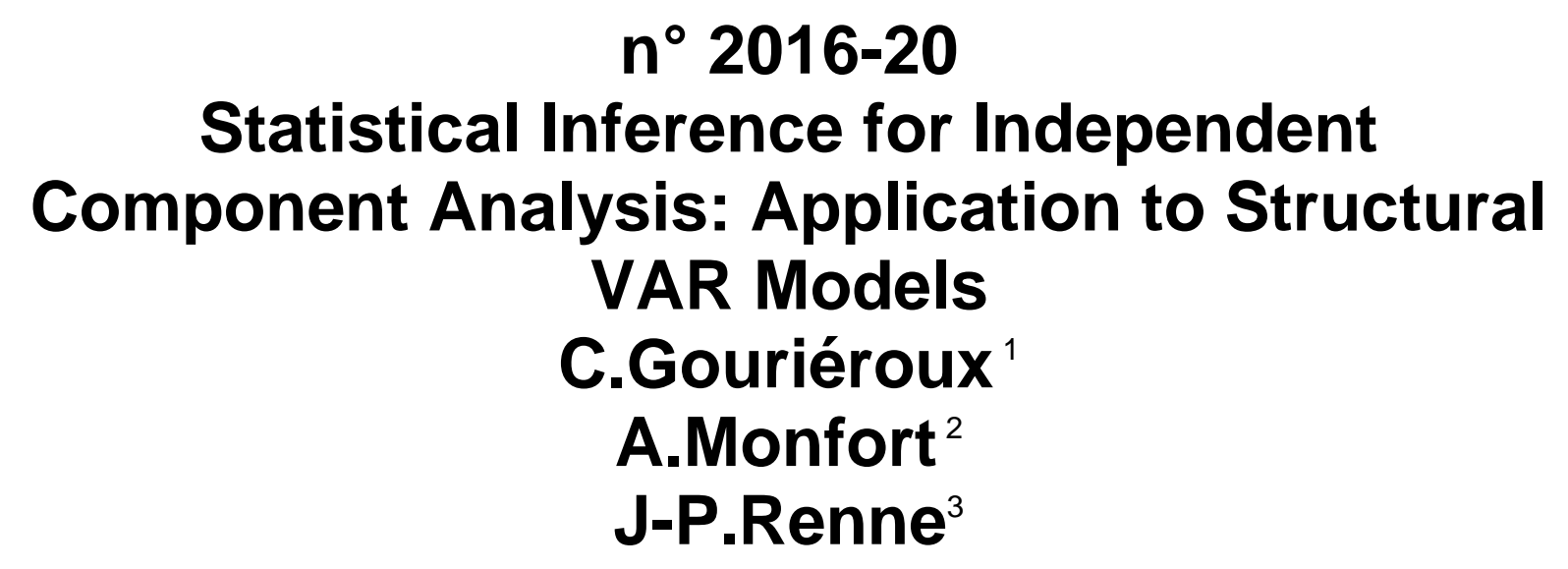

Les documents de travail ne reflètent pas la position du CREST et n'engagent que leurs auteurs. Working papers do not reflect the position of CREST but only the views of the authors.

\footnotetext{
${ }^{1}$ CREST and University of Toronto E-mail: gouriero@ensae.fr

${ }^{2}$ CREST and Banque de France E-mail : Alain.monfort@ensae.fr

${ }^{3}$ University of Lausanne, Faculty of Business and Economics.
} 


\title{
Statistical Inference for Independent Component Analysis: Application to Structural VAR Models
}

\author{
C., GOURIEROUX ${ }^{(1)}$ A., MONFORT ${ }^{(2)}$ and J.-P., RENNE ${ }^{(3)}$ \\ (March 2016, revised version)
}

The first two authors gratefully acknowledge support of the chair LCL: "New Challenges for New Data", of the LABEX: Finance et Croissance Durable, and of the chair ACPR on "Regulation and Systemic Risk".

\footnotetext{
${ }^{1}$ CREST and University of Toronto.

${ }^{2}$ CREST and Banque de France.

${ }^{3}$ University of Lausanne, Faculty of Business and Economics.
} 


\title{
Statistical Inference for Independent Component Analysis: Application to Structural VAR Models
}

\begin{abstract}
The well-known problem of non-identifiability of structural VAR models disappears if the structural shocks are independent and if at most one of them is Gaussian. In that case, the relevant estimation technique is the Independent Component Analysis (ICA). Since the introduction of ICA by Comon (1994), various semi-parametric estimation methods have been proposed for "orthogonalizing" the error terms. These methods include pseudo-maximum likelihood (PML) approaches and recursive PML. However several of these approaches are not consistent and others are significantly subefficient. The aim of our paper is to derive the asymptotic properties of the PML approaches, in particular to study their consistency (or lack of consistency). We conduct Monte Carlo studies exploring the relative performances of these methods. Finally, an application based on real data shows that structural VAR models can be estimated without additional identification restrictions in the non-Gaussian case and that the usual restrictions can be tested.
\end{abstract}

Keywords: Independent Component Analysis, Pseudo-Maximum Likelihood, Identification, Cayley Transform, Structural Shocks, Structural VAR, Impulse Response Functions.

\section{Introduction}

Let us consider $n$ observed variables $Y=\left(y_{1}, \ldots, y_{n}\right)^{\prime}$, which are linear combinations of $n$ independent unobserved sources $\varepsilon=\left(\varepsilon_{1}, \ldots, \varepsilon_{n}\right)^{\prime}$ :

$$
Y=C \varepsilon,
$$

where the components $\varepsilon_{i}$ are zero-mean, and the matrix $C$ is invertible.

$C$ is called the "mixing matrix" and $C^{-1}$ the "demixing matrix". The problem of independent component analysis ${ }^{4}$ (ICA) is to identify $C$ and $\varepsilon$ from the knowledge of $Y$, or, in other words, to consistently estimate $C$ and the distribution of $\varepsilon$, from a large number of observations $Y_{1}, \ldots, Y_{T}$ of vector $Y$.

If $\varepsilon$ is Gaussian, the distribution of $Y$ is also Gaussian, with zero-mean and a variancecovariance matrix $C C^{\prime}$. From the knowledge of the distribution of $Y$, we identify the matrix $C C^{\prime}$, but not matrix $C$ itself. For instance, if $C^{*}=C Q$, where $Q$ is an orthogonal matrix, we

\footnotetext{
${ }^{4}$ In signal processing, the components of $\varepsilon$ are called "sources", the components of $Y$ are called "sensors" and the ICA problem "blind separation of sources". Other terminologies are "sources/mixtures", "signal/mixtures", or "multiple input/multiple output" (MIMO).
} 
have $C^{*} C^{*^{\prime}}=C C^{\prime}$. Thus there is a problem of both local and global identification, since $C$ is identified up to an orthogonal matrix. However the lack of identification almost disappears, if we assume that the components of $\varepsilon$ are independent, not Gaussian. The theorem below has been derived in Eriksson, Koivunen (2004) [see also Comon (1994), Th. 11].

Theorem [Eriksson, Koivunen (2004), Th 3]: Let us consider the independent component model: $Y=C \varepsilon$. Under the following conditions:

i) $C$ is invertible,

ii) The components $\varepsilon_{1}, \ldots, \varepsilon_{n}$ are independent, with at most one Gaussian distribution, then matrix $C$ is identifiable up to the post multiplication by DP, where $P$ is a permutation matrix and $D$ a diagonal matrix with non zero diagonal elements.

In other words $C$ is identifiable up to a permutation of indexes and to signed scaling, $\varepsilon_{i, t} \rightarrow$ $\pm \sigma_{i} \varepsilon_{i, t}, \sigma_{i}>0, i=1, \ldots, n$, say. Thus, for independent non-Gaussian sources, the only cause of local lack of identification is through the positive scaling. The permutation and change in signs of columns of $C$ create a global lack of identification, but not a local one.

The local identification problem, i.e. the possibility of replacing $C$ by $C D$, where $D$ is a diagonal matrix with strictly positive diagonal elements, can be avoided by introducing identification restrictions. Several sets of identification restrictions (SIR) have been considered in the literature. They are:

SIR1 : $c_{i, i}=1, i=1, \ldots, n$ where $c_{i, i}$ is the $i^{\text {th }}$ diagonal term of matrix $C$ [see e.g. Jutten, Herault (1991), Comon, Jutten, Herault (1991), eq. (3), Pham, Garat (1997), p1714, Ilmonen, Paindaveine (2015)].

SIR2 : $c_{i}^{\prime} c_{i}=1, i=1, \ldots, n$, where $c_{i}$ denotes the $i^{\text {th }}$ column of matrix $C$ [see e.g. Comon (1994), Section 5.1, Pham, Garat (1997), p 1714],

or similar sets of identification restrictions written on the diagonal elements $c^{i, i}$, or on the rows $c^{i}, i=1, \ldots, n$, of the demixing matrix $C^{-1}$ :

SIR1* $: c^{i, i}=1, i=1, \ldots, n$,

SIR2* $: c^{i} c^{i^{\prime}}=1, i=1, \ldots, n$ (implicitly used in the one-unit Fast ICA algorithm, see Sections $3.1,3.2)$. 
Stronger conditions can be introduced as in the following set of restrictions:

SIR3 : $C$ is an orthogonal matrix: $C^{\prime} C=I d$ [see e.g. Hyvarinen (1997), eq. 13, Vlassis (2001), eq.23, Hastie, Tibshirani (2002), eq.6].

If the error $\varepsilon$ is standardized $V(\varepsilon)=I d$, these restrictions may imply constraints on the distribution of vector $Y$, such as $V\left(Y_{t}\right)=I d$ for SIR3. This restriction can be asymptotically satisfied if the data are jointly prewhitened.

The restrictions SIR1 and SIR1* have a major drawback, since they implicitly assume that all diagonal elements are different from zero. Thus they exclude a priori some noncausal features between the variables and can bias the impulse response analysis in a dynamic model with independent shocks.

Whenever the independent component model is locally identified, we can expect the existence of consistent semi-parametric estimation methods based on an i.i.d. sample $Y_{1}, \ldots, Y_{T}$. Two types of approaches have been proposed in the literature, that are, pseudo-maximum likelihood (PML) approaches and moment methods. They differ by the form of the objective function, but also by the set of identification restrictions (SIR1-SIR3) that is used. These estimation methods have been introduced mainly in the literature on signal processing and data analysis with a focus on the numerical convergence and computational complexity of the algorithm used to get the estimate [see e.g. Amari, Cardoso (1997), Cardoso (1999), Cardoso, Laheld (1996), Cardoso, Souloumiac (1993), Comon (1994), Sections 4.2, 4.3., Hyvarinen (1997), Section 6, Hyvarinen (1999), Hyvarinen, Oja (1997, 2000), Section 6.1, Vlassis, Motomura (2001)]. As noted in Ilmonen et al. (2012), "In the computer science communities ICA procedures are usually seen as algorithms rather than estimates with their statistical properties." The statistical properties of these estimators, such as their consistency or asymptotic normality, are rarely considered [see Bonhomme, Robin (2009) for an exception in the context of moment methods]. This explains why several standard methods for ICA proposed in the literature or in the softwares are not statistically consistent.

In this paper, we focus on the estimation of independent component models based on PML approaches. More precisely, we carefully examine associated identification issues, we derive the asymptotic statistical behavior of the PML estimators and propose test procedures. We also stress the usefulness of these methods for the identification of structural shocks and the estimation of impulse response functions in non-Gaussian vector autoregressive (VAR) models.

The remaining of this paper is organized as follows. Section 2 presents the pseudo maximum likelihood (PML) approaches for estimating matrix $C$ under SIR3. This section shows that although these methods amounts to maximizing a misspecified log-likelihood function, they 
provide consistent estimators. Then we derive the asymptotic distribution of these PML estimators. In Section 3, we discuss the other PML approaches proposed in the literature. We first show that the one-unit algorithm using identification restrictions as SIR2 or SIR2* provides estimators that are not statistically consistent. Since for large dimension $n$ the optimization of the pseudo likelihood under SIR3 can be numerically cumbersome, we also analyse the recursive PML approaches under SIR3. These approaches compute the estimators of the columns of $C$ in a recursive way. In Section 4, we provide Monte-Carlo experiments as well as an application to real data. While the Monte Carlo experiments compare the finite-sample behavior of the different estimators and evaluate their asymptotic properties, the application on real data stresses the identification of structural shocks and the derivation of impulse response functions. It also shows how our framework makes it possible to test standard over-identification restrictions. Section 5 concludes. Technical results are gathered in appendices.

\section{Pseudo-Maximum Likelihood Approach (under SIR3)}

Let us discuss the consistency and the asymptotic properties of pseudo maximum likelihood estimators of matrix $C$. We first consider the working case of observations such that:

$$
Y_{t}=C_{0} \varepsilon_{t}
$$

where $E_{0}\left(Y_{t}\right)=0, V_{0}\left(Y_{t}\right)=I d, E_{0}\left(\varepsilon_{t}\right)=0, V_{0}\left(\varepsilon_{t}\right)=I d$ and the latent components $\varepsilon_{1, t}, \ldots, \varepsilon_{n, t}$ are assumed both cross-sectionally and serially independent, with unknown true probability density functions (p.d.f.) $f_{i, 0}\left(\varepsilon_{i}\right), i=1, \ldots, n$. In this special framework the $C_{0}$ matrix is orthogonal $C_{0} C_{0}^{\prime}=I d$, which is the set of identification restrictions SIR3, and is identifiable up to a permutation of index $i$ and changes of sign of its columns, if at most one of the true p.d.f. is Gaussian. 5

Then we explain how the results of the working case can be extended to a model of the form:

$$
Y_{t}=a\left(X_{t}, \theta_{0}\right)+S C_{0} \varepsilon_{t}
$$

where $E_{0}\left(Y_{t} \mid X_{t}\right)=a\left(X_{t} ; \theta_{0}\right), V_{0}\left(Y_{t} \mid X_{t}\right)=\Sigma_{0}=S S^{\prime}, E_{0}\left(\varepsilon_{t}\right)=0, V_{0}\left(\varepsilon_{t}\right)=I d$.

\footnotetext{
${ }^{5}$ When the sources are cross-sectionally independent, but serially correlated with distinct spectra, they can be identified by second-order methods, that is, from the knowledge of autocovariances only. This possibility to identify by means of the dynamics of the sources is not considered here. It is the basis of second-order estimation methods as AMUSE [Tong et al. (1990)], or SOBI [Belouchrani et al. (1997)], Gaussian PML written in frequency domain [Pham, Garat (1997), Section 3], or based on canonical correlations [Degerine, Malki (2000)].
} 


\subsection{Pseudo-Maximum Likelihood (PML) estimator}

Let us introduce a set of p.d.f. $g_{i}\left(\varepsilon_{i}\right), i=1, \ldots, n$, and consider the pseudo log-likelihood function:

$$
\log l_{T}(C)=\sum_{t=1}^{T} \sum_{i=1}^{n} \log g_{i}\left(c_{i}^{\prime} Y_{t}\right),
$$

where $c_{i}$ is the $i^{t h}$ column of matrix $C$ (or $c_{i}^{\prime}$ is the $i^{t h}$ row of $C^{-1}$ ). The log-likelihood function (2.3) is computed as if the errors $\varepsilon_{i, t}$ had the p.d.f. $g_{i}\left(\varepsilon_{i}\right)$, and using the fact that $|\operatorname{det} C|=1$, since $C$ is orthogonal. Then a pseudo maximum likelihood (PML) estimator of matrix $C$ maximizes the pseudo log-likelihood function taking into account the condition that $C$ is orthogonal. This optimization problem can be written as:

$$
\begin{gathered}
\hat{C}_{T}=\arg \max _{C} \sum_{t=1}^{T} \sum_{i=1}^{n} \log g_{i}\left(c_{i}^{\prime} Y_{t}\right), \\
\text { s.t. } C^{\prime} C=I d .
\end{gathered}
$$

The optimization problem can also be considered after the elimination of the identification restrictions, that is, after parametrizing the orthogonal matrix $C$. It is known that any orthogonal matrix with no eigenvalue equal to -1 can be written as:

$$
C(A)=(I d+A)(I d-A)^{-1},
$$

where $A$ is a skew symmetric (or antisymmetric) matrix, such that $A^{\prime}=-A$. This is the Cayley's representation of an orthogonal matrix. Moreover, this orthogonal matrix is in a one-to-one relationship with $A$, since we get:

$$
A=(C(A)+I d)^{-1}(C(A)-I d) .
$$

Thus, the PML estimator of matrix $C$ can be alternatively derived as $\hat{C}_{T}=C\left(\hat{A}_{T}\right)$, where:

$$
\hat{A}_{T}=\arg \max _{A} \sum_{t=1}^{T} \sum_{i=1}^{n} \log g_{i}\left[c_{i}(A)^{\prime} Y_{t}\right]
$$

and the optimization is with respect to the parameters characterizing $A$, that are the subdiagonal elements of $A: a_{i, j}, i>j$. 


\subsection{The finite sample first-order conditions (FOC)}

The FOC can be written either on the constrained optimization problem (2.4), or on its parameterized version (2.7). We give in Appendix 1 the closed form expressions of the derivatives of $C(A)$ with respect to $A$, which can be used to derive the FOC for the model written under the parametric form. We focus below on the FOC for problem (2.4).

Let us distinguish the different restrictions on matrix $C$ :

$$
c_{i}^{\prime} c_{j}=0, \quad i<j \text { and } c_{i}^{\prime} c_{i}=1, \quad i=1, \ldots, n
$$

and let us introduce the associated Lagrange multipliers denoted $\lambda_{i, j}=\lambda_{j, i}$, if $i \neq j$, and $\lambda_{i, i} / 2$, when both indices are equal. Then the FOC are:

$$
\left\{\begin{array}{l}
\sum_{t=1}^{T} Y_{t} \frac{d \log g_{i}}{d \varepsilon}\left(\hat{c}_{i}^{\prime} Y_{t}\right)-\sum_{j=1}^{n} \hat{\lambda}_{i, j} \hat{c}_{j}=0, i=1, \ldots, n \\
\hat{c}_{i}^{\prime} \hat{c}_{j}=0, i<j, \hat{c}_{i}^{\prime} \hat{c}_{i}=1, i=1, \ldots, n
\end{array}\right.
$$

We get $n^{2}+n(n-1) / 2+n$ conditions for the $n^{2}+n(n-1) / 2+n$ unknowns, that are the $\hat{c}_{i, j}, \hat{\lambda}_{i, j}, i<j$, and $\hat{\lambda}_{i, i}, i, j=1, \ldots, n$. Premultiplying the first subsystem of (2.8) by $\hat{C}_{T}^{\prime}$ and taking into account the constraints on the orthogonal matrix $\hat{C}$, the finite sample FOC are equivalent to:

$$
\left\{\begin{array}{l}
\sum_{t=1}^{T} \hat{c}_{j}^{\prime} Y_{t} \frac{d \log g_{i}}{d \varepsilon}\left(\hat{c}_{i}^{\prime} Y_{t}\right)-\hat{\lambda}_{i, j}=0, i, j=1, \ldots, n \\
\hat{c}_{i}^{\prime} \hat{c}_{j}=0, i<j, \hat{c}_{i}^{\prime} \hat{c}_{i}=1, \quad i=1, \ldots, n
\end{array}\right.
$$

Since $\hat{\lambda}_{i, j}=\hat{\lambda}_{j, i}$, it is possible to derive from this system the equations giving $\hat{C}_{T}$. They are:

$$
\left\{\begin{array}{l}
\sum_{t=1}^{T} \hat{c}_{j}^{\prime} Y_{t} \frac{d \log g_{i}}{d \varepsilon}\left(\hat{c}_{i}^{\prime} Y_{t}\right)-\sum_{t=1}^{T} \hat{c}_{i}^{\prime} Y_{t} \frac{d \log g_{j}}{d \varepsilon}\left(\hat{c}_{j}^{\prime} Y_{t}\right)=0, \quad i<j, \\
\hat{c}_{i}^{\prime} \hat{c}_{j}=0, i<j, \hat{c}_{i}^{\prime} \hat{c}_{i}=1, \quad i=1, \ldots, n .
\end{array}\right.
$$

Thus the FOC of the constrained optimization problem (2.4) lead to a subsystem giving the estimate of $C$.

Let us denote by $\mathscr{P}(M)$ the set of matrices obtained by permuting and changing the signs of the columns of $M$. It is worth noting that, if the function $g_{i}$ are different and not even, the value 
of the objective function $\sum_{t=1}^{T} \sum_{i=1}^{n} \log g_{i}\left(c_{i}^{\prime} Y_{t}\right)$ obtained by taking $C$ equal to an element of $\mathscr{P}\left(\hat{C}_{T}\right)$, different from $\hat{C}_{T}$, will be different and therefore smaller than the one obtained with $\hat{C}_{T}$. On the other hand, in the extreme case where all the $g_{i}$ 's are equal and even, all the elements of $\mathscr{P}\left(\hat{C}_{T}\right)$ will provide a maximum.

\subsection{Consistency}

To derive conditions for the consistency of the PML estimators when $T$ goes to infinity (and $n$ is fixed), we have to consider the associated asymptotic optimization problem and the asymptotic FOC. We have already made the following assumptions on the sources $\varepsilon_{t}$ 's:

\section{Assumption A.1}

i) The shocks $\varepsilon_{t}$ are i.i.d. with $E_{0}\left(\varepsilon_{t}\right)=0$ and $V_{0}\left(\varepsilon_{t}\right)=I d$.

ii) The components $\varepsilon_{1, t}, \ldots, \varepsilon_{n, t}$ are mutually independent.

In addition we make the following assumption on the p.d.f. of the sources:

\section{Assumption A.2}

i) The functions $\log g_{i}, i=1, \ldots, n$, are twice continuously differentiable.

ii) $\sup _{C: C^{\prime} C=I d}\left|\sum_{i=1}^{n} \log g_{i}\left(c_{i}^{\prime} y\right)\right| \leq h(y)$, where $E_{0}[h(Y)]<\infty$.

From Assumption A.1 and A.2 ii), we know that the finite sample objective function: $Q_{T}(C)=\frac{1}{T} \sum_{t=1}^{T} \sum_{i=1}^{n} \log g_{i}\left(c_{i}^{\prime} Y_{t}\right)$ tends almost surely uniformly to the asymptotic one, which is $Q_{\infty}(C)=E_{0}\left[\sum_{i=1}^{n} \log g_{i}\left(c_{i}^{\prime} Y_{t}\right)\right]$.

Moreover, the parameter set, that is, the set of orthogonal matrices, is compact. Then the uniform integrability in Assumption A.2 ii) implies the uniform convergence of $Q_{T}$ towards $Q_{\infty}$, and the convergence of the optimizers of $Q_{T}$ to the set of optimiser of $Q_{\infty}$ [Jennrich (1969), Gourieroux, Monfort (1995), vol 2, chapter 24]. Finally the latter optimizers can be analyzed by means of the asymptotic FOC. This approach is followed below. 
The asymptotic optimization problem is:

$$
\max _{C} L_{\infty}(C)=\max _{C} \operatorname{plim}_{T \rightarrow \infty} \frac{1}{T} \log l_{T}(C) \equiv \max _{C} \sum_{i=1}^{n} E_{0}\left[\log g_{i}\left(c_{i}^{\prime} Y_{t}\right)\right]
$$

s.t. $c_{i}^{\prime} c_{j}=0, i<j, c_{i}^{\prime} c_{i}=1, i, j=1, \ldots, n$ with Lagrange multipliers $\lambda_{i, j, 0}, \lambda_{i, i, 0} / 2$. The asymptotic FOC are:

$$
\left\{\begin{array}{l}
E_{0}\left[Y_{t} \frac{d \log g_{i}}{d \varepsilon}\left(c_{i}^{\prime} Y_{t}\right)\right]-\sum_{j=1}^{n} \lambda_{i, j} c_{j}=0, \quad i=1, \ldots, n, \\
c_{i}^{\prime} c_{j}=0, i<j, c_{i}^{\prime} c_{i}=1, \quad i, j=1, \ldots, n
\end{array}\right.
$$

By premultiplying the set of equations by $c_{k}^{\prime}$, by using the conditions of orthogonal matrix and the equality $\lambda_{i, j}=\lambda_{j, i}$, the asymptotic FOC imply:

$$
\left\{\begin{array}{l}
\lambda_{i, j}=E_{0}\left[c_{j}^{\prime} Y_{t} \frac{d \log g_{i}}{d \varepsilon}\left(c_{i}^{\prime} Y_{t}\right)\right]=E_{0}\left[c_{i}^{\prime} Y_{t} \frac{d \log g_{j}}{d \varepsilon}\left(c_{j}^{\prime} Y_{t}\right)\right]=\lambda_{j, i}, \quad i \neq j \\
\lambda_{i, i}=E_{0}\left[c_{i}^{\prime} Y_{t} \frac{d \log g_{i}}{d \varepsilon}\left(c_{i}^{\prime} Y_{t}\right)\right], \quad i=1, \ldots, n
\end{array}\right.
$$

We deduce the following property:

Proposition 1 For any element $C$ of $\mathscr{P}\left(C_{0}\right)$, and the associated $\varepsilon_{i, t}$ 's, the values $C, \lambda_{i, j, 0}=0$, $i<j, \lambda_{i, i, 0}=E_{0}\left[\varepsilon_{i, t} \frac{d \log g_{i}\left(\varepsilon_{i, t}\right)}{d \varepsilon}\right], i=1, \ldots, n$ are solutions of the asymptotic FOC.

Proof Indeed replacing the $c_{i}$ 's by their true values, we get:

$$
\lambda_{i, j, 0}=E_{0}\left[\varepsilon_{j, t} \frac{d \log g_{i}\left(\varepsilon_{i, t}\right)}{d \varepsilon}\right]=E_{0}\left[\varepsilon_{i, t} \frac{d \log g_{j}\left(\varepsilon_{j, t}\right)}{d \varepsilon}\right]=\lambda_{j, i, 0} .
$$

Then, by the independence of $\varepsilon_{i, t}, \varepsilon_{j, t}$ for $i \neq j$, we get:

$$
E_{0}\left[\varepsilon_{j, t} \frac{d \log g_{i}\left(\varepsilon_{i, t}\right)}{d \varepsilon}\right]=E_{0}\left(\varepsilon_{j, t}\right) E_{0}\left[\frac{d \log g_{i}\left(\varepsilon_{i, t}\right)}{d \varepsilon}\right]=0,
$$

since $\varepsilon_{j, t}$ is zero-mean. The conclusion follows.

\section{QED}

We deduce a necessary identification assumption. 


\section{Assumption A.3 Identification from the asymptotic FOC.}

The only solutions of the system of equations:

$$
\left\{\begin{array}{l}
E_{0}\left[c_{j}^{\prime} Y_{t} \frac{d \log g_{i}}{d \varepsilon}\left(c_{i}^{\prime} Y_{t}\right)\right]=0, i \neq j \\
C^{\prime} C=I d
\end{array}\right.
$$

are the elements of $\mathscr{P}\left(C_{0}\right)$, which is the set of matrices obtained by permutation and sign change of the columns of $C_{0}$.

As seen in the next proposition, Assumption A.3 implies restrictions on the true distribution of $Y_{t}$ as well as on the choice of the pseudo p.d.f. $g_{i}$ 's.

\section{Proposition 2}

a) If at least two components of $Y_{t}$ have the Gaussian distribution $N(0,1)$, are independent from each other and independent from the other components, then Assumption A.3 cannot be satisfied.

b) If at least two pseudo p.d.f. $g_{i}$ and $g_{j}$ are Gaussian $N(0,1)$, then Assumption A.3 cannot be satisfied.

\section{Proof}

a) Let us assume, without loss of generality, that $Y_{1, t}$ and $Y_{2, t}$ have the $N(0,1)$ distribution and are independent. Let $C$ be an orthogonal matrix satisfying A.3 and $C^{*}$ the orthogonal matrix obtained from $C$ by permuting its first two rows. It is easily seen that $C^{*}$ also satisfies A.3. Indeed, for any column $c_{i}$ of $C$ and the corresponding column $c_{i}^{*}$ of $C$ we have

$$
\begin{aligned}
& c_{i}^{\prime} Y_{t}=c_{i, 1} Y_{1, t}+c_{i, 2} Y_{2, t}+\Sigma_{k \geq 2} c_{i, k} Y_{k, t}, \\
& c_{i}^{*^{\prime} Y_{t}}=c_{i, 2} Y_{1 t}+c_{i, 1} Y_{2, t}+\Sigma_{k \geq 2} c_{i, k} Y_{k, t},
\end{aligned}
$$

and, since $c_{i, 1} Y_{1, t}+c_{i, 2} Y_{2, t}$ and $c_{i, 2} Y_{1, t}+c_{i, 1} Y_{2, t}$ have the same distribution $N\left(0, c_{i, 1}^{2}+c_{i, 2}^{2}\right)$, the result follows.

b) We have $\frac{\partial \log g_{i}}{d \varepsilon}\left(c_{i}^{\prime} Y_{t}\right)=-c_{i}^{\prime} Y_{t}$ and $\frac{d \log g_{i}\left(c_{j} Y_{t}\right)}{d \varepsilon}=-c_{j}^{\prime} Y_{t}$. Therefore the corresponding $(i, j)$ condition of Assumption A.3 is: $E_{0}\left(c_{j}^{\prime} Y_{t} c_{i}^{\prime} Y_{t}\right)=E_{0}\left(c_{i}^{\prime} Y_{t} c_{j}^{\prime} Y_{t}\right)$, which is satisfied for any $C$ and for any true distribution of $Y_{t}$. 
Even if Assumption A.3 is satisfied, we are not sure that a matrix $C$ of $\mathscr{P}\left(C_{0}\right)$ corresponds to a maximum of the asymptotic optimization problem. To check this property, we can consider a second-order expansion of $L_{\infty}(C)$ in a neighbourhood of the true value. It is shown in Appendix A.2.1 that the asymptotic objective function is locally concave under the following assumption:

\section{Assumption A.4 Local concavity.}

The asymptotic objective function is locally concave in a neighbourhood of a matrix $C$ of $\mathscr{P}\left(C_{0}\right)$ if and only if

$$
E_{0}\left[\frac{d^{2} \log g_{i}\left(\varepsilon_{i, t}\right)}{d \varepsilon^{2}}+\frac{d^{2} \log g_{j}\left(\varepsilon_{j, t}\right)}{d \varepsilon^{2}}-\varepsilon_{j, t} \frac{d \log g_{j}\left(\varepsilon_{j, t}\right)}{d \varepsilon}-\varepsilon_{i, t} \frac{d \log g_{i}\left(\varepsilon_{i, t}\right)}{d \varepsilon}\right]<0, \forall i<j
$$

where $\varepsilon_{i, t}$ is the $i^{\text {th }}$ component of the $\varepsilon_{t}$ associated with this particular element $C$ of $\mathscr{P}\left(C_{0}\right)$.

This condition is in particular satisfied under the following set of conditions derived in Hyvarinen (1997), Th. 1 [see also Hyvarinen, Karhunen, Oja (2001), Th. 8.1]: ${ }^{6}$

$$
E_{0}\left[\frac{d^{2} \log g_{i}\left(\varepsilon_{i, t}\right)}{d \varepsilon^{2}}-\varepsilon_{i, t} \frac{d \log g_{i}\left(\varepsilon_{i, t}\right)}{d \varepsilon}\right]<0, i=1, \ldots, n
$$

This set of conditions is sufficient but not necessary. Hyvarinen, Karhunen, Oja (2001) have exhibited a couple of distributions that is such that either one or the other satisfy the inequality (2.12) as long as $E_{0}\left(\varepsilon_{i, t}\right)=0$, and $E_{0}\left(\varepsilon_{i, t}^{2}\right)=1$. These distributions are the Hyperbolic secant and the subgaussian distributions reported in Table $1 .^{7}$

For a given set of pseudo density functions in a given order $g_{1}, \ldots, g_{n}$, the value of the asymptotic criterion $\sum_{i=1}^{n} E_{0}\left[\log g_{i}\left(c_{i}^{\prime} Y_{t}\right)\right]$ for a given element $C$ of $\mathscr{P}\left(C_{0}\right)$ is:

$$
\sum_{i=1}^{n} E_{0}\left[\log g_{i}\left(\varepsilon_{i, t}\right)\right]
$$

where $\varepsilon_{i, t}$ is the $i^{t h}$ component of the $\varepsilon_{t}$ associated with this particular element $C$ of $\mathscr{P}\left(C_{0}\right)$.

\footnotetext{
${ }^{6}$ Note that, if the pseudo distribution $g_{i}$ is $N(0,1)$ or even $N\left(m_{i}, \sigma_{i}^{2}\right)$, the left hand side of the inequality is equal to zero, for any true distribution of $\varepsilon_{i, t}$ satisfying $E_{0}\left(\varepsilon_{i, t}\right)=0$ and $E_{0}\left(\varepsilon_{i, t}^{2}\right)=1$.

${ }^{7}$ This statement is easily checked by using the third and fourth columns of this table to compute the expectation appearing on the left-hand side of Inequality (2.12) (and using $E_{0}\left(\varepsilon_{i, t}^{2}\right)=1$ ).
} 


\section{Assumption A.5 Distinct distributions.}

The pseudo distributions $g_{i}$, as well as the true distributions of the $\varepsilon_{i, t}$, are different and asymmetric.

If Assumption A.4 is satisfied for a $C \in \mathscr{P}\left(C_{0}\right)$, such a matrix will provide a local maximum of the asymptotic criterion. If Assumption A.5 is also satisfied, then the values of the asymptotic criterion at these local maxima will be in general different. Therefore, in that case, the global maximum will be reached by a unique element of $\mathscr{P}\left(C_{0}\right)$. For the sake of notational simplicity, let us denote by $C_{0}$ the value of $C$ giving this global maximum. We have the following consistency result: ${ }^{8}$

Proposition 3 Under Assumptions A.1-A.5, the PML estimator of C exists asymptotically and is a consistent estimator of $C_{0}$.

Thus the misspecification of pseudo-distributions $g_{i}$ has no effect on the consistency of these specific PML estimators. This is easily understood when we consider the asymptotic FOC in (2.11). They simply correspond to zero moment conditions written on:

$$
c_{j}^{\prime} Y_{t} \frac{d \log g_{i}}{d \varepsilon}\left(c_{i}^{\prime} Y_{t}\right)-c_{i}^{\prime} Y_{t} \frac{d \log g_{j}}{d \varepsilon}\left(c_{j}^{\prime} Y_{t}\right), \quad i<j
$$

The consistency result is still valid if $g_{i}$ is not a p.d.f., but the interpretation as misspecified ML is more appealing.

\subsection{Asymptotic distribution of the PML estimator}

The asymptotic accuracy of the PML estimator depends on the choice of the pseudo p.d.f.. Its asymptotic distribution is derived in Appendix 4. Again, let us denote by $C_{0}$ the unique value of $C$ giving the unique global maximum of the asymptotic criterion under the conditions given above.

Proposition 4 Under Assumptions A.1-A.5, the PML estimator $\hat{C}_{T}$ of $C_{0}$ is asymptotically normal, with speed of convergence $1 / \sqrt{T}$. The asymptotic variance-covariance matrix of vec $\sqrt{T}\left(\hat{C}_{T}-C_{0}\right)$ is $A^{-1}\left[\begin{array}{cc}\Omega & 0 \\ 0 & 0\end{array}\right]\left(A^{\prime}\right)^{-1}$, where $A$ and $\Omega$, given in Appendix 4, are square

\footnotetext{
${ }^{8}$ If the global maximum of the asymptotic criterion is reached on a subset $E_{0}$ of $\mathscr{P}\left(C_{0}\right)$, the PML estimator will converge to $E_{0}$, that is $\hat{C}_{T}-C_{0, T}$ will converge to zero, where $C_{0, T}=\underset{C \in E_{0}}{\operatorname{Argmin}} d\left(\hat{C}_{T}, C\right), d$ being any distance.
} 
matrices of respective sizes $n^{2}$ and $\frac{n(n-1)}{2}$.

The previous result implies that the asymptotic Gaussian distribution has a support of dimension $\frac{n(n-1)}{2}$, as expected since an orthogonal matrix must satisfy $\frac{n(n+1)}{2}$ constraints.

For illustration, let us consider the bivariate case $n=2$. The asymptotic expansion of the FOC shows that:

$$
\sqrt{T}\left(\begin{array}{c}
\hat{c}_{1}-c_{1,0} \\
\hat{c}_{2}-c_{2,0}
\end{array}\right)=\left[\begin{array}{cc}
\gamma_{1,2} c_{2,0}^{\prime} & \gamma_{2,1} c_{1,0}^{\prime} \\
c_{10}^{\prime} & c_{20}^{\prime} \\
c_{10}^{\prime} & 0 \\
0 & c_{20}^{\prime}
\end{array}\right]^{-1}\left[\begin{array}{l}
Z \\
0 \\
0 \\
0
\end{array}\right]
$$

where

$$
\begin{aligned}
\gamma_{i, j}= & E_{0}\left[\frac{d^{2} \log g_{i}\left(\varepsilon_{i, t}\right)}{d \varepsilon^{2}}\right]-E_{0}\left[\varepsilon_{j, t} \frac{d \log g_{j}\left(\varepsilon_{j, t}\right)}{d \varepsilon}\right], \\
Z \sim & N\left(0, w^{2}\right), \\
w^{2}= & E_{0}\left\{\left[\frac{d \log g_{1}\left(\varepsilon_{1, t}\right)}{d \varepsilon}\right]^{2}\right\}+E_{0}\left\{\left[\frac{d \log g_{2}\left(\varepsilon_{2, t}\right)}{d \varepsilon}\right]^{2}\right\} \\
& -2 E_{0}\left[\varepsilon_{1, t} \frac{d \log g_{1}\left(\varepsilon_{1, t}\right)}{d \varepsilon}\right] E_{0}\left[\varepsilon_{2, t} \frac{d \log g_{2}\left(\varepsilon_{2, t}\right)}{d \varepsilon}\right] .
\end{aligned}
$$

The expression of the asymptotic variance can be simplified in the bivariate case (see Appendix 5.1). We get:

$$
V_{a s}\left[\sqrt{T}\left(\operatorname{vec} \hat{C}_{T}-\operatorname{vec} C_{0}\right)\right]=\frac{w^{2}}{\left(\gamma_{1,2}+\gamma_{2,1}\right)^{2}}\left(\begin{array}{cc}
c_{2,0} c_{2,0}^{\prime} & -c_{2,0} c_{1,0}^{\prime} \\
-c_{1,0} c_{2,0}^{\prime} & c_{1,0} c_{1,0}^{\prime}
\end{array}\right)
$$

This closed form expression facilitates the consistent estimation of the asymptotic variance of $\hat{C}_{T}$. Indeed, from the PML estimates $\hat{C}_{T}$ we deduce the approximated errors $\hat{\varepsilon}_{t}=\hat{C}_{T}^{\prime} Y_{t}$. Therefore $\gamma_{i, j}$ and $w^{2}$ are consistently estimated by replacing their theoretical expectations by their sample counterparts and the errors $\varepsilon$ by their approximations $\hat{\varepsilon}$. For instance, we can take:

$$
\hat{\gamma}_{i, j}=\frac{1}{T} \sum_{t=1}^{T} \frac{d^{2} \log g_{i}\left(\hat{\varepsilon}_{i, t}\right)}{d \varepsilon^{2}}-\frac{1}{T} \sum_{t=1}^{T}\left[\hat{\varepsilon}_{j, t} \frac{d \log g_{i}\left(\hat{\varepsilon}_{j, t}\right)}{d \varepsilon}\right] .
$$


For $n=2$, the elements of $C$ generate a manifold of dimension 1 (see Appendix 5). Thus the asymptotic variance-covariance matrix is of rank 1. It has been suggested in Pham, Garat (1997), Section 2.B., to also consider the asymptotic distribution of transformations of $\hat{C}_{T}$ such as: ${ }^{9}$

$$
\hat{\Delta}_{T}=I d-C^{-1} \hat{C}_{T}=I d-C^{\prime} \hat{C}_{T}
$$

We show in Appendix 5.2 that:

$$
V_{a s}\left[\sqrt{T} v e c \hat{\Delta}_{T}\right]=\frac{\omega^{2}}{\left(\gamma_{1,2}+\gamma_{2,1}\right)^{2}}\left[\begin{array}{cccc}
0 & 0 & 0 & 0 \\
0 & 1 & 1 & 0 \\
0 & 1 & 1 & 0 \\
0 & 0 & 0 & 0
\end{array}\right]
$$

Thus, after this transformation the asymptotic accuracy of $\hat{\Delta}_{T}$ no longer depends on matrix $C$, but only on the distributional properties of the sources and of the pseudo p.d.f..

Finally, the multiplicative factor function $\omega^{2} /\left(\gamma_{1,2}+\gamma_{2,1}\right)^{2}$ differs from the multiplicative factors derived in Hyvarinen (1997), eq. 15, or in Pham, Garat (1997), where the restrictions on $C$ required for identification do not seem to have been fully taken into account in their derivations.

Going back to the general case we see that the asymptotic accuracy of the PML estimator depends on the choice of the pseudo p.d.f.. Since the ML estimator is asymptotically efficient, we immediately deduce the following corollary [see also Pham, Garat (1997)]:

Corollary 1 The asymptotic accuracy of the PML estimator is maximal if $g_{i}$, the pseudo p.d.f. of $\varepsilon_{i, t}$, is equal to its true p.d.f.

The corollary above raises the following two comments:

i) The practice of selecting a pseudo p.d.f. as far as possible to a Gaussian distribution, for instance by maximizing a distance to Gaussianity such as the negentropy, or an approximation of the negentropy, by third and fourth-order cumulants is suboptimal, ${ }^{10}$ especially when the true distribution is close to Gaussian.

ii) The asymptotic efficiency for the estimation of parameter $C$ could be reached in two steps by an adaptive estimation approach. In a first step $C$ is estimated by a non efficient

\footnotetext{
${ }^{9}$ For expository purpose we have changed the definition of the so-called contamination coefficients initially defined as $\hat{\Delta}_{T}=I d-\hat{C}_{T}^{-1} C$.

${ }^{10}$ See Kaiser (1958) for an early version of such an idea, or the choice $g_{i}(y)=\operatorname{sech}^{2}(y) / 2$, whose associated score function is $2 \tanh (y)$ introduced in the informax algorithm [Bell, Sejnowski (1995) or Hyvarinen, Karhunen, Oja (2001), p111, 222-223].
} 
PML approach. The corresponding estimate is used to compute the residuals as: $\hat{\varepsilon}_{t}=\hat{C}_{T}^{\prime} Y_{t}$, $t=1, \ldots, T$. Next the approximated sources $\hat{\varepsilon}_{i, t}, t=1, \ldots, T$ can be used to estimate nonparametrically the densities $f_{i, 0}, i=1, \ldots, n$. In a second step the PML approach is reapplied with $g_{i}=\hat{f}_{i}, i=1, \ldots, n$, where $\hat{f}_{i}$ is a consistent functional estimator of $f_{i, 0}$.

\subsection{Testing procedures}

Let us now consider the problem of testing that the true value of $C$ belongs to $\mathscr{P}_{0}$, where $\mathscr{P}_{0}$ is the set of orthogonal matrices obtained by permuting and changing the signs of the columns of a given orthogonal matrix $C_{0}$ (i.e. $\mathscr{P}_{0}=\mathscr{P}\left(C_{0}\right)$ ). We denote by $C_{j, 0}, j \in J$, the elements of $\mathscr{P}_{0}$.

The null hypothesis $H_{0}$ stating that the true value of $C$ belongs to $\mathscr{P}_{0}$ is not standard since it is a finite union of simple hypotheses $H_{0, j}=\left(C=C_{j, 0}\right)$.

A first testing procedure consists in defining the Wald statistics $\hat{\xi}_{j, T}, j \in J$ :

$$
\hat{\xi}_{j, T}=T\left[\operatorname{vec} \hat{C}_{T}-\operatorname{vec} C_{j, 0}\right]^{\prime} \hat{A}_{T}^{\prime}\left[\begin{array}{rr}
\hat{\Omega}_{T}^{-1} & 0 \\
0 & 0
\end{array}\right] \hat{A}_{T}\left[\operatorname{vec} \hat{C}_{T}-\operatorname{vec} C_{j, 0}\right]
$$

$\hat{A}_{T}$ and $\hat{\Omega}_{T}$ being consistent estimators of the matrices $A$ and $\Omega$ defined in Proposition 4 and Appendix 4. Since the dimension of the asymptotic distribution of $\sqrt{T}\left[\operatorname{vec} \hat{C}_{T}-\operatorname{vec} C_{j, 0}\right]$ is $\frac{1}{2} n(n-1)$, the asymptotic distribution of $\hat{\xi}_{j, T}$ under $H_{0, j}$ is $\chi^{2}\left(\frac{1}{2} n(n-1)\right)$.

Then we define:

$$
\hat{\xi}_{T}=\min _{j \in J} \hat{\xi}_{j, T}
$$

as the test statistic for the null hypothesis of interest $H_{0}$. Under the null hypothesis, $\hat{C}_{T}$ converges to $C_{j_{0}, 0}$, say. By the asymptotic properties of the Wald statistics for simple hypotheses, we have that:

$$
\hat{\xi}_{j_{0}, T} \stackrel{D}{\rightarrow} \chi^{2}\left(\frac{n(n-1)}{2}\right)
$$

and $\hat{\xi}_{j, T} \rightarrow \infty$ if $j \neq j_{0}$.

Under the null hypothesis, $\hat{\xi}_{T}=\min _{j} \hat{\xi}_{j, T}$ is asymptotically equal to $\hat{\xi}_{j_{0}, T}$ (since, for $j \neq$ $j_{0}, \hat{\xi}_{j_{0}, T}$ goes to $\left.+\infty\right)$ and its asymptotic distribution, $\chi^{2}\left(\frac{1}{2} n(n-1)\right)$, does not depend on $j_{0}$. Therefore $\hat{\xi}_{T}$ is asymptotically a pivotal statistic for the null hypothesis $H_{0}$ and the test of critical region $\hat{\xi}_{T} \geq \chi_{1-\alpha}^{2}\left(\frac{1}{2} n(n-1)\right)$ is of asymptotic level $\alpha$ and is consistent.

The second testing method is the following. Let us first define $C_{0, T}=\operatorname{Argmin} d\left(\hat{C}_{T}, C\right)$ where $d$ is any distance, for instance the Euclidian one. 
Under the null hypothesis $H_{0}:\left(C \in \mathscr{P}_{0}\right), \hat{C}_{T}$ converges almost surely to an element of $\mathscr{P}_{0}$ denoted by $C_{j_{0}, 0}$ and it is also the case for $C_{0, T}$ since, asymptotically, we have $C_{0, T}=$ $C_{j_{0}, 0}$. Moreover, $\sqrt{T}\left(\hat{C}_{T}-C_{0, T}\right)=\sqrt{T}\left(\hat{C}_{T}-C_{j_{0}, 0}\right)+\sqrt{T}\left(C_{j_{0}, 0}-C_{0, T}\right)$ and, since $C_{0, T}$ is almost surely asymptotically equal to $C_{j_{0}, 0}$, the asymptotic distribution of $\sqrt{T}\left(\hat{C}_{T}-C_{0, T}\right)$ under $H_{0}$ is the same as that of $\sqrt{T}\left(\hat{C}_{T}-C_{j_{0}, 0}\right)$. This implies that

$$
\tilde{\xi}_{T}=T\left[\operatorname{vec} \hat{C}_{T}-\operatorname{vec} C_{0, T}\right]^{\prime} \hat{A}_{T}^{\prime}\left[\begin{array}{cc}
\hat{\Omega}_{T}^{-1} & 0 \\
0 & 0
\end{array}\right] \hat{A}_{T}\left[\operatorname{vec} \hat{C}_{T}-\operatorname{vec} C_{0, T}\right]
$$

is asymptotically distributed as $\chi^{2}\left(\frac{1}{2} n(n-1)\right)$ under $H_{0}$.

An advantage of this second method is that it necessitates the computation of only one Wald test statistic.

\subsection{Structural VARs, structural shocks and impulse response functions}

The results of the subsections above can be used to derive consistent semi-parametric estimators in models of the type:

$$
Y_{t}=a\left(X_{t} ; \theta\right)+S C \varepsilon_{t},
$$

where $E\left(Y_{t} \mid X_{t}\right)=a\left(X_{t} ; \theta\right), V\left(Y_{t} \mid X_{t}\right)=\Sigma, C$ is an orthogonal matrix, $S$ is any matrix satisfying $S S^{\prime}=\Sigma$ (it can for instance be the matrix resulting from the Cholesky decomposition of $\Sigma$ with positive diagonal entries) and $\left(\varepsilon_{t}\right)$ satisfies Assumption A.1.

The parameters $\theta, \Sigma$ can be estimated by nonlinear least squares: $\hat{\theta}_{T}$ is the solution of:

$$
\hat{\theta}_{T}=\arg \min _{\theta} \sum_{t=1}^{T}\left\|Y_{t}-a\left(X_{t} ; \theta\right)\right\|^{2}
$$

Then a consistent estimator of $\Sigma$ is:

$$
\hat{\Sigma}_{T}=\frac{1}{T}\left[Y_{t}-a\left(X_{t} ; \hat{\theta}_{T}\right)\right]\left[Y_{t}-a\left(X_{t} ; \hat{\theta}_{T}\right)\right]^{\prime}
$$

These first-step estimators are used to compute standardized OLS residuals:

$$
\hat{u}_{t}=\hat{S}_{T}^{-1}\left[Y_{t}-a\left(X_{t} ; \hat{\theta}_{T}\right)\right]
$$

where $\hat{S}_{T}$ is such that $\hat{S}_{T} \hat{S}_{T}^{\prime}=\hat{\Sigma}_{T}$. The orthogonal matrix $C$ is finally estimated by applying a PML approach on the series of residuals $\hat{u}_{t}$. 
This consistent estimation approach can be applied to dynamic models. In particular it can be used to identify independent shocks in a structural vector autoregressive (SVAR) model [see e.g. Chen, Choi, Escanciano (2012), Moneta et al. (2013), Gourieroux, Monfort (2014)]. In this case the explanatory variables $X_{t}$ are lagged endogenous variables and the model of interest is:

$$
\Phi(L) Y_{t}=S C \varepsilon_{t}
$$

with $\Phi(L)=I d-\Phi_{1} L-\ldots-\Phi_{p} L^{p}, L$ being the lag operator and the roots of $\operatorname{det} \Phi(L)$ being outside the unit circle. In this context, the independent components $\varepsilon_{j, t}$ of $\varepsilon_{t}$ are called "structural" shocks. Inverting $\Phi(L)$ gives the infinite moving average representation:

$$
Y_{t}=\sum_{k=0}^{\infty} \Theta_{k} S C \varepsilon_{t-k}, \text { with } \Theta_{0}=I d .
$$

The impulse response function (IRF) of $Y_{i, t}$ to a unitary shock on $\varepsilon_{j, t}$ is the sequence:

$$
I R F_{i, j}(k)=\Theta_{i, k} S c_{j}
$$

where $\Theta_{i, k}$ is the $i^{t h}$ row of $\Theta_{k}$. The estimation results in the estimated IRF:

$$
\widehat{I R F}_{i, j}(k)=\hat{\Theta}_{i, k} \hat{S}_{T} \hat{c}_{j}
$$

The fact that $\lim _{T \rightarrow \infty} \hat{C}_{T}$ is one or another element of $\mathscr{P}\left(C_{0}\right)$ is totally harmless. Indeed the ordering of the components of $\varepsilon_{t}$ is arbitrary; it is just a problem of labelling of these components. Similarly it is always possible to rename $-\varepsilon_{j, t}$ as $\varepsilon_{j, t}$ and to change the sign of $c_{j}$ accordingly.

The economic interpretation of the structural independent shocks $\varepsilon_{j, t}$ can be based on the shapes of the impulse response function $\left\{\widehat{I R F}_{i, j}(k), k=0,1,2, \ldots,\right\}$ for $1, \ldots, n$, that are perfectly identified in our context, without any additional conditions (see the application of Section 4).

The assumption of independence of the components of $\varepsilon_{t}$ is particularly important in the context of "structural shocks". Indeed, if this assumption is not satisfied, it is impossible to shock one component of $\varepsilon_{t}$ without affecting the others. In the economic literature the structural shocks $\varepsilon_{j, t}$ are, more or less explicitly, assumed to be Gaussian [see Moneta et al. (2013) for an exception]. In this context the structural shocks are not identified and identifying restrictions are required, such as restrictions on the short run impact of the shocks [see e.g. Bernanke (1986), Sims (1986), Rubio-Ramirez, Waggoner, Zha (2010)], or on the long run impacts [see 
e.g. Blanchard, Quah (1989), Faust, Leeper (1997), Erceg, Guerrieri, Gust (2005), Christiano, Eichenbaum, Vigfusson (2006)], as well as on the sign of some impulse response functions [see e.g. Uhlig (2005), Chari, Kehoe, McGrattan (2008), Mountford, Uhlig (2009)]. By contrast, in our non Gaussian framework such additional restrictions imply overidentification and become testable. This new situation is illustrated in Section 4.

\section{Links with the PML Literature on ICA}

In Section 2, we have derived the asymptotic properties of a PML approach, namely its consistency and its asymptotic normality using the constraints of orthogonal matrix $C$ to solve the identification issue.

There exist other PML estimation methods proposed in the literature on ICA and on blind separation of sources. They differ by the identification restrictions that are used, by the possible introduction of auxiliary parameters in the pseudo-log likelihood function, by the global or recursive nature of the optimization problem and by the possible prewhitening of the observed data. Since the literature mainly deals with signal processing and data analysis, there is a focus on the numerical convergence and computational complexity of the algorithm used to optimize the pseudo log-likelihood function. A few papers consider the asymptotic distribution of PML or recursive PML estimators [see e.g. Pham, Garat (1997), Hyvarinen (1997), Ilmonen et al. (2012)], but these papers give no proof of the statistical consistency of the PML estimators. This explains why among the PML methods proposed in the literature and in the softwares several are not statistically consistent. This might also explain practical suggestions such as "In real world problems, it is useful to apply several ICA algorithms, because they may reveal different IC's from the data" [Hyvarinen, Karhunen, Oja (2001), p286]. The aim of this section is to review these alternative PML approaches, to discuss their consistency (or their lack of consistency), and to derive their asymptotic distributional properties.

\subsection{Identification by row specific constraints}

Let us consider the ICA model:

$$
Y_{t}=C_{0} \varepsilon_{t}
$$

with the standard assumptions: $C_{0}$ is invertible, the variables $\varepsilon_{1, t}, \ldots, \varepsilon_{n, t}$ are independent, zero mean, and the $\varepsilon_{i, t}, t=1, \ldots, T$, have the same distribution $f_{i, 0}\left(\varepsilon_{i}\right)$. But we do not impose $V\left(\varepsilon_{i, t}\right)=1$. Whereas, in Section 2, we have solved the identification issue by assuming an orthogonal matrix $C$, that is by imposing specific and cross restrictions on the columns $c_{i}$, 
$i=1, \ldots, n$, of matrix $C$, namely $c_{i}^{\prime} c_{i}=1$ and $c_{i}^{\prime} c_{j}=0$, the identification issue might also be solved by imposing restrictions only on the rows of $C^{-1}$, that are the SIR2* restrictions: ${ }^{11}$

$$
c^{i}\left(c^{i}\right)^{\prime}=1
$$

The PML optimization problem becomes:

$$
\begin{gathered}
\hat{B}_{T}=\arg \max _{B} \sum_{t=1}^{T} \sum_{i=1}^{n} \log g_{i}\left(b_{i}^{\prime} Y_{t}\right), \\
\text { s.t. } b_{i}^{\prime} b_{i}=1, \quad i=1, \ldots, n,
\end{gathered}
$$

where $B$ denotes the matrix parameter whose rows are $b_{i}^{\prime}, i=1, \ldots, n$.

This problem is numerically simple, since it is equivalent to $n$ optimization problems, which can be solved independently:

$$
\hat{b}_{i, T}=\arg \max _{b_{i}} \sum_{t=1}^{T} \log g_{i}\left(b_{i}^{\prime} Y_{t}\right) \quad \text { s.t. } b_{i}^{\prime} b_{i}=1 .
$$

Such optimization problems are called "one-unit algorithms" in the ICA literature [see e.g. Hyvarinen, Oja (2000), Hyvarinen, Karhunen, Oja (2001), Section 8.3].

The following property shows that $\hat{b}_{i, T}^{\prime}$ does not converge to a row of $\left(C_{0}\right)^{-1}$ if $C_{0}$ is not orthogonal; moreover, if $C_{0}$ is orthogonal, a repeated implementation of (3.3) does not guarantee to obtain consistent estimators of all the rows of $\left(C_{0}\right)^{-1}$ or, equivalently, all the columns of $C_{0}$.

Proposition 5 The one-unit algorithms provide statistically consistent estimators neither of $C^{-1}$, nor of $C$, for any $C \in \mathscr{P}\left(C_{0}\right)$.

Proof When the true matrix $C_{0}$ is not necessarily orthogonal, the expected interpretation of the pseudo-parameter $B_{0}=\lim _{T \rightarrow \infty} \hat{B}_{T}$ is $C_{0}^{-1}$. Thus we focus below on this expected interpretation.

i) Let us consider the asymptotic first-order conditions corresponding to the optimization

\footnotetext{
${ }^{11} C_{0}$ is identifiable up to a transformation of the form $C_{0} D P=C_{0}^{*}$, where $D$ is a diagonal matrix and $P$ a permutation matrix. This implies $C_{0}^{*-1}=P^{\prime} D^{-1} C_{0}^{-1}$. The constraints $c^{i}\left(c^{i}\right)^{\prime}=1$ for $C_{0}$ can be written $e_{i}^{\prime} C_{0}^{-1}\left(C_{0}^{-1}\right)^{\prime} e_{i}=1$ $\forall i$, where $e_{i}$ is the $i^{t h}$ selection vector. In this case, we have $e_{i}^{\prime} C_{0}^{*-1}\left(C_{0}^{*-1}\right)^{\prime} e_{i}=e_{i}^{\prime} P^{\prime} D^{-1} C_{0}^{-1} C_{0}^{-1^{\prime}} D^{-1} P e_{i}$, Noting $e_{j}=P e_{i}$ and $d_{j}$ the $j^{t h}$ diagonal term of $D^{-1}$, we get $e_{i}^{\prime} C_{0}^{*-1}\left(C_{0}^{*-1}\right)^{\prime} e_{i}=d_{j}^{2}$ and the constraints on $C_{0}^{*}$ imposes $d_{j}^{2}=1$, or $d_{j}= \pm 1$. In other words, the set of matrices observationally equivalent to $C_{0}$ is again $\mathscr{P}\left(C_{0}\right)$.
} 
problem (3.2). They are:

$$
\left\{\begin{array}{l}
E_{0}\left[Y_{t} \frac{d \log g_{i}}{d \varepsilon}\left(b_{i}^{\prime} Y_{t}\right)\right]-\lambda_{i, i} b_{i}=0, \quad i=1, \ldots, n \\
b_{i}^{\prime} b_{i}=1, \quad i=1, \ldots, n
\end{array}\right.
$$

We can eliminate the Lagrange multipliers and deduce the equations satisfied by the pseudo-true values only:

$$
E_{0}\left[Y_{t} \frac{d \log g_{i}}{d \varepsilon}\left(b_{i}^{\prime} Y_{t}\right)\right]-E_{0}\left[b_{i}^{\prime} Y_{t} \frac{d \log g_{i}}{d \varepsilon}\left(b_{i}^{\prime} Y_{t}\right)\right] b_{i}=0, \quad i=1, \ldots, n
$$

Let us now check whether the solutions in $b_{i}$ of this system can be the transposes of the rows $c_{0}^{i}, i=1, \ldots, n$, of matrix $C_{0}^{-1}$. System (3.4) becomes:

$$
\begin{aligned}
& E_{0}\left[C_{0} \varepsilon_{t} \frac{d \log g_{i}}{d \varepsilon}\left(c_{0}^{i} Y_{t}\right)\right]-E_{0}\left[c_{0}^{i} Y_{t} \frac{d \log g_{i}}{d \varepsilon}\left(c_{0}^{i^{\prime}} Y_{t}\right)\right]\left(c_{0}^{i}\right)^{\prime}=0, \quad i=1, \ldots, n \\
\Leftrightarrow & E_{0}\left[C_{0} \varepsilon_{t} \frac{d \log g_{i}\left(\varepsilon_{i, t}\right)}{d \varepsilon}\right]-E_{0}\left[\varepsilon_{i, t} \frac{d \log g_{i}\left(\varepsilon_{i, t}\right)}{d \varepsilon}\right]\left(c_{0}^{i}\right)^{\prime}=0, \quad i=1, \ldots, n \\
\Leftrightarrow & \left.E_{0}\left[\varepsilon_{i, t} \frac{d \log g_{i}\left(\varepsilon_{i, t}\right.}{d \varepsilon}\right)\right]\left(c_{i, 0}-\left(c_{0}^{i}\right)^{\prime}\right)=0, \quad i=1, \ldots, n,
\end{aligned}
$$

by using the independence between the components of error $\varepsilon_{t}$ and the fact that these components are zero-mean. We deduce that a necessary condition for this one-unit algorithm to provide a consistent estimator of $C_{0}^{-1}$ is:

$$
c_{i, 0}=\left(c_{0}^{i}\right)^{\prime}, \quad i=1, \ldots, n,
$$

that is the orthogonality of the true matrix $C_{0}$.

ii) Let us now assume that the true matrix $C_{0}$ is orthogonal. We know from the discussion above that the asymptotic FOC are satisfied by $b_{i}=c_{i, 0}$. However, it is also seen that this PML estimator is not statistically consistent in general. Indeed let us choose, as it is standard in the literature, the same pseudo p.d.f. for all indexes $i$. Then the different optimization problems indexed by $i$ have the same solution, that is $\hat{b}_{i, T}=\hat{b}_{T}$, independent on $i$. If they are consistent, their limits are the same $c_{0}$, say, and the pseudo-true value of matrix $B$ is $\left(c_{0}, c_{0}, \ldots, c_{0}\right)$. Matrix $B$, which is therefore noninvertible, cannot be equal to $C_{0}^{-1}$. 
Of course different independent components may be estimated if we change the pseudo p.d.f. in several optimizations of the objective function or if we run the algorithm using different starting points. Such an approach is rather ad-hoc and does not ensure to find the total number of linearly independent components, contrary to multi-unit methods such as the PML approach developed in Section 2. This is the reason why this approach is usually replaced by the recursive PML described in Section 3.4.

\subsection{One-unit algorithm with row specific constraints and introduction of auxiliary parameters}

For the same reason, there is a lack of consistency for more sophisticated one-unit PML approaches. Let us consider the most favorable case of an orthogonal $C_{0}$ matrix. We denote by $\sigma_{i, 0}^{2}$ the variance of $\varepsilon_{i, t}$ and by $D_{0}$ the diagonal matrix whose diagonal terms are the $\sigma_{i, 0}^{2}$ 's. The variance-covariance matrix of $Y_{t}$ is $C_{0} D_{0} C_{0}^{\prime}$, which is not constrained. It has been suggested to jointly consider the estimation of matrix $C_{0}$, with row specific restrictions on $C^{-1}$, or equivalently with column restrictions on $C$, and of the variances $\sigma_{i, 0}^{2}, i=1, \ldots, n$. The PML estimator is defined on $C^{-1}=C^{\prime}$ by:

$$
\begin{gathered}
\left(\hat{C}_{T}, \operatorname{vec}\left(\hat{\sigma}_{T}^{2}\right)\right)=\underset{C, \operatorname{vec}\left(\sigma^{2}\right)}{\arg } \sum_{t=1}^{T} \sum_{i=1}^{n}\left[\log g_{i}\left(\frac{c_{i}^{\prime} Y_{t}}{\sigma_{i}}\right)-\frac{1}{2} \log \sigma_{i}^{2}\right], \\
\text { s.t. } c_{i}^{\prime} c_{i}=1, \quad i=1, \ldots, n,
\end{gathered}
$$

which is equivalent to $n$ optimizations of smaller dimension considered independently:

$$
\left(\hat{c}_{i, T}, \hat{\sigma}_{i, T}^{2}\right)=\underset{c_{i}, \sigma_{i}^{2}}{\arg \max } \sum_{t=1}^{T}\left[\log g_{i}\left(\frac{c_{i}^{\prime} Y_{t}}{\sigma_{i}}\right)-\frac{1}{2} \log \sigma_{i}^{2}\right], \quad \text { s.t. } c_{i}^{\prime} c_{i}=1 .
$$

It is checked in Appendix A.3.1, that the asymptotic FOC are satisfied by values $c_{i, 0}, \sigma_{i, 0}^{2 *}$, where $c_{i, 0}$ is the true value of the $i^{t h}$ column of $C$ and $\sigma_{i, 0}^{2 *}$ differs from the true value $\sigma_{i, 0}^{2}$. Thus, from the FOC, we might expect $\hat{c}_{i, T}$ to be a consistent estimator of $c_{i, 0}$. However, this approach is not consistent in general for the same reason as in the second part of the proof of Proposition 5. We therefore have the following result:

Proposition 6 The one-unit algorithm with auxiliary volatility parameter does not provide a statistically consistent estimator of $C_{0}$, even if $C_{0}$ is an orthogonal matrix. Moreover $\hat{\sigma}_{i, T}^{2}$ does not converge to the true variance $\sigma_{i, 0}^{2}$ of $\varepsilon_{i, t}$. 


\subsection{Jacobian adjusted PML with row specific constraints and auxiliary parameters}

The pseudo-likelihood used in optimization (3.3) is misspecified since the pseudo p.d.f. does not correspond to the true p.d.f., but also since we have not taken into account the Jacobian effect. The Jacobian adjusted PML is the solution of:

$$
\begin{aligned}
& \left(\hat{B}_{T}, \operatorname{vec} \hat{\sigma}_{T}^{2}\right)=\arg \max _{C, \operatorname{vec}\left(\sigma^{2}\right)} \sum_{t=1}^{T} \sum_{i=1}^{n}\left[\log g_{i}\left(\frac{\left.b_{i}^{\prime} Y_{t}\right)}{\sigma_{i}}\right)-\frac{1}{2} \log \sigma_{i}^{2}+\log |\operatorname{det} B|\right], \\
& \text { s.t. } b_{i}^{\prime} b_{i}=1, \quad i=1, \ldots, n .
\end{aligned}
$$

This form of objective function has been considered in Pham, Garat (1997), Section 2.A, but without taking into account explicitly the constraints $b_{i}^{\prime} b_{i}=1, \ldots, n$ in the FOC. ${ }^{12}$ Moreover, contrary to the title of their Section 2: "The ML approach for white sources", they do not really study the properties of the associated PML estimator, but modify the FOC to get covariance restrictions [see their equation (2.1)]. As in Sections 3.1-3.2, the estimator $\hat{B}_{T}-$ solution of the optimization problem (3.7) - is not a consistent estimator of $C_{0}^{-1}$ (see Appendix A.3.2), except if $C_{0}$ is orthogonal.

\subsection{Recursive PML approach (under SIR3)}

We have seen in Subections 3.1-3.3 that the one-unit identification restrictions SIR2 or SIR2* are not sufficient to get the consistency of the PML estimator of $C_{0}$ (or $C_{0}^{-1}$ ), even if the pseudolikelihood is Jacobian adjusted. Let us now come back to the set of identification restrictions SIR3.

\section{i) The recursive scheme}

The identification constraints of orthogonality of $C$ can also be introduced in a recursive optimization scheme. Let us consider the same assumptions as in Section 2. In particular $C_{0}$ is orthogonal. We can apply a recursive PML approach, called deflation based Fast ICA in the literature [see e.g. Ollila (2010), Reyhani et al. (2012), Ilmonen et al. (2012), Miettinen et al. (2014)]. The recursive PML estimator is derived by a succession of simplified optimization problems.

More precisely at step $i$, the recursive PML estimators $\hat{c}_{1}, \ldots, \hat{c}_{i-1}$ have already been derived

\footnotetext{
${ }^{12}$ Even if these restrictions are mentioned p1713: $\hat{C}_{T}$ is defined "up to a scaling factor for each of its column".
} 
and the recursive PML estimator $\hat{c}_{i}$ of $c_{i}$ is defined as the solution of:

$$
\hat{c}_{i}=\arg \max _{c_{i}} \sum_{t=1}^{T} \log g_{i}\left(c_{i}^{\prime} Y_{t}\right), \quad \text { s.t. }: \quad c_{i}^{\prime} c_{i}=1, \quad c_{i}^{\prime} \hat{c}_{j}=0, \quad j=1, \ldots, i-1,
$$

for $i=2, \ldots, n$. For $i=1$, the only constraint is $c_{1}^{\prime} c_{i}=1$.

\section{ii) The Gaussian case}

This recursive PML approach has been initially proposed by analogy with principal component analysis (PCA) [see e.g. Lawley, Maxwell (1971), Anderson (1984) for PCA]. PCA is based on a PML approach with Gaussian pseudo-distributions. Taking the standard Gaussian densities for all the densities $g_{i}$ in formula (2.3), the optimization problem of Section 2 becomes:

$$
\begin{gathered}
\max _{C}-\sum_{t=1}^{T} \sum_{i=1}^{n}\left(c_{i}^{\prime} Y_{t}\right)^{2} \\
\text { s.t. } C^{\prime} C=I d .
\end{gathered}
$$

The objective function can also be written as:

$$
\begin{aligned}
-\sum_{t=1}^{T} \sum_{i=1}^{n} c_{i}^{\prime} Y_{t} Y_{t}^{\prime} c_{i} & =-\sum_{i=1}^{n}\left[c_{i}^{\prime} \sum_{t=1}^{T} Y_{t} Y_{t}^{\prime} c_{i}\right]=-\operatorname{Tr}\left[C^{\prime} \sum_{t=1}^{T} Y_{t} Y_{t}^{\prime} C\right] \\
& =-\operatorname{Tr}\left[\sum_{t=1}^{T} Y_{t} Y_{t}^{\prime} C C^{\prime}\right](\text { by commuting within the Trace operator) } \\
& =-\operatorname{Tr}\left(\sum_{t=1}^{T} Y_{t} Y_{t}^{\prime}\right)\left(\text { since } C C^{\prime}=I d\right) .
\end{aligned}
$$

Thus the objective function takes the same value for all orthogonal matrices $C$. This is the well-known identification problem of matrix $C$ in the Gaussian framework (see the introduction). Then the recursive Gaussian PML is used in PCA to find an easily interpretable matrix $C$. Indeed the solution of the recursive PML approach is the sequence of unit norm eigenvectors of $\sum_{t=1}^{T} Y_{t} Y_{t}^{\prime}$ associated with the eigenvalues ranked in decreasing order (assuming that there is no multiple eigenvalue).

\section{iii) Recursive vs global optimization PML estimators}

When the pseudo p.d.f.'s are not Gaussian, the PML estimator of Section 2 and the recursive PML estimator are not necessarily equal in finite sample. For instance let us consider $n=2$ and 
parametrize matrix $C$ as: ${ }^{13}$

$$
C=\left(\begin{array}{ll}
\cos \theta & -\sin \theta \\
\sin \theta & \cos \theta
\end{array}\right)
$$

The PML estimator of $\theta$ is the solution of

$$
\max _{\theta} \sum_{t=1}^{T}\left\{\log g_{1}\left(y_{1, t} \cos \theta+y_{2, t} \sin \theta\right)+\log g_{2}\left(-y_{1, t} \sin \theta+y_{2, t} \cos \theta\right)\right\},
$$

whereas the recursive PML estimator of $\theta$ is the solution of

$$
\max _{\theta} \sum_{t=1}^{T}\left[\log g_{1}\left(y_{1, t} \cos \theta+y_{2, t} \sin \theta\right)\right] .
$$

It is easily seen that the solutions of these optimization problems differ in finite sample (even up to a permutation of the columns and to a change of sign of the columns of $C$ ). They also have different asymptotic properties. Indeed the conditions of local concavity differ (see Assumption $\tilde{A} .4$ below). They are, respectively:

$$
\begin{aligned}
& \text { - } \\
& E_{0}\left[\frac{d^{2} \log g_{1}\left(\varepsilon_{1}\right)}{d \varepsilon^{2}}+\frac{d^{2} \log g_{2}\left(\varepsilon_{2}\right)}{d \varepsilon^{2}}-\varepsilon_{1} \frac{d \log g_{1}\left(\varepsilon_{1}\right)}{d \varepsilon}-\varepsilon_{2} \frac{d \log g_{2}\left(\varepsilon_{2}\right)}{d \varepsilon}\right]<0, \\
& \text { and } \quad E_{0}\left[\frac{d^{2} \log g_{1}\left(\varepsilon_{1}\right)}{d \varepsilon^{2}}-\varepsilon_{1} \frac{d \log g_{1}\left(\varepsilon_{1}\right)}{d \varepsilon}\right]<0 .
\end{aligned}
$$

The previous identification Assumptions A.3-A.4 are replaced by (see Appendices A.3.3 and A.2.2 for the justification):

Assumption Ã.3 For any $i=1, \ldots, n-1$, the system:

$$
\begin{aligned}
& E_{0}\left\{\frac{d \log g_{i}}{d \varepsilon}\left(c_{i}^{\prime} Y_{t}\right)\left[\sum_{j=i}^{n} c_{j, 0} \varepsilon_{j, t}-c_{i}^{\prime} Y_{t} c_{i}-\Sigma_{j<i} \varepsilon_{j, t} c_{j, 0}\right]\right\}=0, \\
& c_{i}^{\prime} c_{i}=1, c_{i}^{\prime} c_{j, 0}=0, j<i, i, j=1, \ldots, n,
\end{aligned}
$$

has the (essentially) ${ }^{14}$ unique solution $c_{i, 0}$.

\footnotetext{
${ }^{13}$ This parametrization is valid for an orthogonal matrix $C$ such that $\operatorname{det} C=1$.

${ }^{14}$ That is $c_{i, 0}$ is one of the remaining columns (or its opposite) of a matrix of $\mathscr{P}\left(C_{0}\right)$ containing the columns $c_{j, 0}, j<i$, (or their opposite), once these columns have been eliminated.
} 


\section{Assumption A.4 Local concavity.}

The asymptotic objective function is locally concave in a neighbourhood of $C_{0}$ if and only if

$$
E_{0}\left[\frac{d^{2} \log g_{i}\left(\varepsilon_{i, t}\right)}{d \varepsilon^{2}}-\varepsilon_{i, t} \frac{d \log g_{i}\left(\varepsilon_{i, t}\right)}{d \varepsilon}\right]<0, i=1, \ldots, n-1 .
$$

\section{iv) Behaviour of the recursive PML estimator}

We prove in Appendix 3.3 that the asymptotic FOC are satisfied by the true values. Moreover, if the true distributions of the $\varepsilon_{i, t}$ are different and asymmetric and if the pseudo distributions $g_{i}$ are asymmetric, the optimal values of the asymptotic criterion at step $i\left(E_{0}\left(\log g_{i}\left(\varepsilon_{i, t}\right)\right)\right.$ where $\varepsilon_{i, t}$ is associated with a particular choice of $C$ in $\mathscr{P}\left(C_{0}\right)$ ) will change if this choice changes and, therefore, the global maximum of this asymptotic criterion will be reached by a unique element denoted by $C_{0}$ for the sake of simplicity. Under the previous assumption we get the following result:

Proposition 7 Let us assume that the true matrix $C_{0}$ is orthogonal.

i) Even for the same set of pseudo distributions, the PML and recursive PML estimators of $C_{0}$ under SIR3 generally differ in finite sample.

ii) Under Assumptions A. A-A.A the PML and recursive PML estimators of $C_{0}$ are consistent.

iii) Even for the same set of pseudo distributions, the asymptotic distributions of the PML and recursive PML estimators generally differ.

The FOC of the finite sample optimization problem (3.8) are:

$$
\left\{\begin{array}{l}
\sum_{t=1}^{T} Y_{t} \frac{d \log g_{i}}{d \varepsilon}\left(\hat{c}_{i}^{\prime} Y_{t}\right)-\sum_{j=1}^{i} \hat{\lambda}_{i, j} \hat{c}_{j}=0, \quad i=1, \ldots, n, \\
\hat{c}_{i}^{\prime} \hat{c}_{j}=0, \quad j<i, \quad \hat{c}_{i}^{\prime} \hat{c}_{i}=1, \quad i=1, \ldots, n,
\end{array}\right.
$$

where $\hat{\lambda}_{i, j}, j<i$ (resp. $\left.\hat{\lambda}_{i, i} / 2\right)$ is the estimated Lagrange multiplier associated with the restriction $c_{i}^{\prime} \hat{c}_{j}=0, j<i\left(\right.$ resp. $\left.c_{i}^{\prime} c_{i}=1\right)$. Note that at the $n^{\text {th }}$ iteration $\hat{c}_{n}$ is (essentially) characterized by the orthogonality restrictions and $g_{n}$ has no impact on the asymptotic distribution of the recursive PML estimator while it has an impact on the asymptotic distribution of the PML estimator. 
As for deriving system (2.9) of FOC for the PML estimator, we can premultiply the first subsystem by $\hat{C}_{T}^{\prime}$. We get:

$$
\sum_{t=1}^{T} \hat{c}_{j}^{\prime} Y_{t} \frac{d \log g_{i}}{d \varepsilon}\left(\hat{c}_{i}^{\prime} Y_{t}\right)-\hat{\lambda}_{i, j}=0, j \leq i
$$

Then we can substitute this expression of the Lagrange multiplier in the system to get:

$$
\begin{aligned}
& \sum_{t=1}^{T}\left[Y_{t} \frac{d \log g_{i}}{d \varepsilon}\left(\hat{c}_{i}^{\prime} Y_{t}\right)-\sum_{j=1}^{i}\left(\hat{c}_{j}^{\prime} Y_{t} \frac{d \log g_{i}}{d \varepsilon}\left(\hat{c}_{i}^{\prime} Y_{t}\right) \hat{c}_{j}\right)\right]=0, i=1, \ldots, n, \\
\Longleftrightarrow & \sum_{t=1}^{T}\left\{\frac{d \log g_{i}}{d \varepsilon}\left(\hat{c}_{i} Y_{t}\right)\left[Y_{t}-\sum_{j=1}^{i} \hat{c}_{j}^{\prime} Y_{t} \hat{c}_{j}\right]\right\}=0, i=1, \ldots, n .
\end{aligned}
$$

This system is easily solved recursively.

Additional asymptotic distributional properties of recursive PML estimators have been derived in Ilmonen et al. (2012), Theorem 2.2. and Miettinen et al (2014). In particular it has been realized that Assumption $\tilde{A} .3$ is often not satisfied when the same functions $\frac{d \log g_{i}}{d \varepsilon}$, independent of $i$, are introduced in the different steps [see Miettinen et al. (2014), p2].

\section{Applications}

\subsection{Monte Carlo exercises}

This subsection presents the results of a Monte-Carlo exercise where we use the PML approaches presented above (under SIR3). After having specified a 2-dimensional orthogonal mixing matrix $C_{0}$, we simulate samples of i.i.d. zero-mean and unit-variance shocks $\varepsilon_{1, t}$ and $\varepsilon_{2, t}$ and we pre-multiply $\varepsilon_{t}=\left[\varepsilon_{1, t}, \varepsilon_{2, t}\right]^{\prime}$ by $C_{0}$ to get $Y_{t}$ vectors. We denote by $\mathscr{D}_{j}$ the distribution used to draw $\varepsilon_{j, t}$. For each pair of generating distributions $\left(\mathscr{D}_{1}, \mathscr{D}_{2}\right), N=5000$ samples are generated, each one being of length $T$. We consider different sample sizes $T=200,500$ and 5000 . The $i^{t h}$ simulated sample is denoted by $\left\{Y_{t}^{(i)}\right\}_{t \in[1, T]}, i=1, \ldots, 5000$. In our simulations, we use different distributions $\mathscr{D}_{j}$. More precisely, we use Student distributions with different degrees of freedom as well as a hyperbolic secant distribution [see Baten (1934)]. The logarithms of the associated p.d.f. as well as the analytical expressions of their first two derivatives are reported in Table 1.

For each simulated sample, we apply different PLM approaches to estimate matrix $C_{0}$ : the 
PML approach of Section 2 (with different sets of pseudo distributions $\left(g_{1}, g_{2}\right)$ ) as well as the recursive PML approach of Subsection 3.4 (with different pseudo distributions $g_{1}$ ). ${ }^{15}$

Because $C_{0}$ is a 2-dimensional orthogonal matrix, it depends on a single parameter. Hence, in our exercise, we focus on the estimation of $c_{1,1}$, where this parameter is set at $\cos (-\pi / 5)=$ 0.809. ${ }^{16}$ Table 2 presents summary statistics associated with the distributions of the estimators $\widehat{c}_{1,1}$ of $c_{1,1}$, for the different generating distributions $\left(\mathscr{D}_{1}, \mathscr{D}_{2}\right)$, estimation techniques and sample sizes $T$. The computation of these statistics is based on the set of obtained estimators $\left\{\widehat{c}_{1,1}^{(i)}\right\}_{i \in[1,5000]}$. Figures 1 displays the kernel-based distributions of $\widehat{c}_{1,1}$ for $T=500$.

The results suggest that the PML estimates of $c_{1,1}$ tend to be negatively biased (Panel (a) in Table 2). As expected, the bias is smaller for larger samples. For all sample sizes, nonrecursive PML estimates are more accurate than recursive ones: for instance, for 500-period (respectively 5000-period) samples, root-mean-squared errors (RMSEs) are twice (respectively 3 times) lower for non-recursive PML estimates than for recursive ones. This can also be seen on Figure 1 by comparing the upper and lower panels. Noteworthy is the fact that, for nonrecursive PMLs, the choice of the pseudo-distributions has a mild impact on the estimators accuracy. In particular, when the pseudo distributions $\left(g_{1}, g_{2}\right)$ do not coincide with $\left(\mathscr{D}_{1}, \mathscr{D}_{2}\right)$, the data-generating ones, we do not observe a significant increase in the RMSEs of $c_{1,1}$ estimates.

Based on the same simulations and estimations, we conduct another exercise to assess the small-sample validity of the asymptotic distributions of $C$ 's estimators. For each simulated sample $i \in[1,5000]$, we compute the asymptotic covariance matrix as detailed in Appendix 4. Then we use the asymptotic standard deviation estimate of $c_{1,1}$, denoted by ${\widehat{\sigma_{1,1}}}^{(i)}$, to derive a confidence interval of level $\alpha$ for $c_{1,1}$; this interval is $\left[{\widehat{c_{1,1}}}^{(i)}-\phi_{\alpha / 2}{\widehat{\sigma_{1,1}}}^{(i)},{\widehat{c_{1,1}}}^{(i)}+\phi_{\alpha / 2}{\widehat{\sigma_{1,1}}}^{(i)}\right]$, where $\phi_{\alpha}$ is the $\alpha^{\text {th }}$ quantile of the standard normal distribution. Eventually, we compute the fraction of estimations for which $c_{1,1}$ lies in the interval. Let us denote this fraction by $f_{\alpha}$. If the distribution of the finite-sample estimates of $c_{1,1}$ were equal to the asymptotic one, we would have $\alpha \equiv f_{\alpha}$.

Table 3 shows the results of this exercise. Even for relatively short sample size $(T=200)$, the asymptotic distributions of the estimators are good approximations of their small-sample distributions. Indeed, in most cases, the fractions $f_{\alpha}$ are close to the confidence levels $\alpha$. In particular, the asymptotic approximations do not appear to be worse in cases where the pseudo-

\footnotetext{
${ }^{15}$ For the recursive approach a single pseudo-distribution is needed since in the bivariate case $C_{0}$ depends on a single parameter and is therefore identified at the end of the first step.

${ }^{16}$ The mixing matrix $C_{0}$ is such that $\operatorname{Vec}\left(C_{0}\right)=[0.809,-0.588,0.588,0.809]^{\prime}$. Recall that $C$ is identified up to sign and permutation of its columns. Therefore, the estimator $\widehat{c_{1,1}}$ is an estimate of either $c_{1,1},-c_{1,1}, c_{1,2}$ or $-c_{1,2}$. In order to deal with this, after each estimation, we look for the transformation of $\hat{C}_{T}$ (out of 4 ) that is the closest to $C$ (in the sense that the sum of the squared deviations between the elements of $C$ and those of the transformed matrix $\hat{C}_{T}$ is the lowest), see discussion in Section 2.5.
} 
distributions do not coincide with the true generating ones.

\subsection{Structural VARs}

In this subsection, we show how independent component analysis can be used to identify structural shocks and their associated impulse response functions (IRFs) in the context of vector autoregressive (VAR) models. ${ }^{17}$ For the sake of illustration, we consider a small-scale VAR model involving three dependent variables stacked in vector $Y_{t}$ (say), that are the inflation $\left(\pi_{t}\right)$, the economic activity $\left(y_{t}\right)$ and the nominal short-term interest rate $\left(r_{t}\right)$. In that context, the structural shocks we aim at identifying are as follows: a monetary-policy shock, a demand shock and a supply shock.

Let us denote by $X_{t}$ the set of information made of the past values of $Y_{t}$, that is $\left\{Y_{t-1}, Y_{t-2}, \ldots\right\}$, and of exogenous variables $\left\{Z_{t}, Z_{t-1}, \ldots\right\}$. Using the notations of Subsection 2.6, in the (reducedform) VAR model:

$$
Y_{t}=a\left(X_{t} ; \theta\right)+u_{t}
$$

where the mean of $Y_{t}$ conditional on $X_{t}$ is given by $a\left(X_{t} ; \theta\right)=\mu+\sum_{i=1}^{p} \Phi_{i} Y_{t-1}+\Gamma Z_{t}$, and the $u_{t}$ 's are serially independent, with zero mean and variance-covariance matrix $\Sigma$ conditional on $X_{t}$.

Our dataset covers the period from 1959:IV to 2015:I at the quarterly frequency ( $T=224)$. All data are extracted from the Federal Reserve Economic Database (FRED). We consider two different measures of economic activity extensively used in the literature, that are the output gap and the unemployment gap, respectively. ${ }^{18}$ Inflation is calculated as the change in the logarithm of the GDP deflator. The change in the logarithm of oil prices is added as an exogenous variable in each of the three VAR equations. ${ }^{19}$ Following the Akaike criteria, we select VAR specifications with six lags. ${ }^{20}$ Parameters $\mu, \Phi_{i}, \Gamma$ and $\Sigma$ are consistently estimated by OLS. Jarque-Bera tests support the hypothesis of non-normality for all residuals, opening the door to the ICA machinery.

\footnotetext{
${ }^{17}$ Comprehensive presentations of VAR models and reviews of this literature are provided by, e.g., Canova (1994), Watson (1994), Stock and Watson (2001), or Lütkepohl (2005).

${ }^{18}$ The output gap is computed as the deviation of the natural logarithm of real GDP (mnemonic GDPC1) from a measure of the log potential GDP (mnemonic GDPPOT). The unemployment gap is computed as the difference between the observed unemployment rate (mnemonic UNRATE) and the natural rate of unemployment (mnemonic NROU).

${ }^{19}$ Sims (1992), or Leeper, Sims and Zha (1996) have shown that the introduction of commodity prices in VAR models help to eliminate the positive response of prices to contractionary monetary policy shocks.

${ }^{20}$ The Hannan-Quinn and Schwartz criteria point to a lower number of lags ( 3 and 2 respectively) whatever the chosen measure of real activity. However, portmanteau tests suggest that for such low numbers of lags, residuals are strongly auto-correlated.
} 
We want to estimate the orthogonal matrix $C$ such that $u_{t}$ is equal to $S C \varepsilon_{t}$, where $S$ is the lower triangular matrix resulting from the Cholesky decomposition of $\Sigma$ with positive diagonal entries and the components of $\varepsilon_{t}$ are independent, zero-mean with unit variance. Since the $u_{t}$ 's are not observed, the PML approach will be applied on standardized VAR residuals, the latter being obtained by pre-multiplying the residuals $\hat{u}_{t}$, i.e. $Y_{t}-a\left(X_{t} ; \hat{\theta}_{T}\right)$, by $\hat{S}_{T}^{-1}$. The pseudo density functions we use are those of three distinct and asymmetric mixtures of Gaussian distributions. $^{21}$

Once $C$ has been estimated, it remains to associate the structural shocks (monetary-policy, supply or demand) with the different components of $\varepsilon_{t}$. To that purpose, we rely on basic economic theory stating that contractionary monetary-policy shocks are expected to have a (short-term and medium-term) negative impact on real activity and on inflation. Moreover, contrary to the demand shock, the supply shock is expected to have (short-term and mediumterm) influences of opposite signs on economic activity and on inflation. Figure 2 displays the IRFs resulting from the ICA approach (see the black solid lines). For both VAR models, associated with the two measures of economic activity, there is only one of the three shocks that is such that an increase in the short-term rate is accompanied by a decrease in both inflation and economic activity: ${ }^{22}$ this shock corresponds to the third row of IRFs, and could be seen as a contractionary monetary-policy shock. Out of the two remaining shocks, one has influences of opposite signs on economic activity and on inflation (second row of IRFs). Because this shock has a positive impact on economic activity, it could be seen as an expansionary supply shock. The remaining shock could be seen as an expansionary demand shock (first row of IRFs).

Table 4 displays the results of the PML estimation of matrix $C$ for the two VAR models. The left-hand side (respectively right-hand side) of the table corresponds to the model where economic activity is proxied by the output gap (resp. the unemployment gap). Asymptotic standard deviations are also reported.

It is natural to compare our results with those stemming from the standard "recursive" identification approach based on specific short-run restrictions (SRRs). This approach, originally due to Sims $(1980 a, b)$ is based on the assumptions that (a) the covariance matrix of the structural shocks is the identity matrix, (b) the $k^{\text {th }}$ structural shock does not contemporaneously affects the first $k-1$ endogenous variables and (c) the contemporaneous effect of the $k^{\text {th }}$ structural shock on the $k^{\text {th }}$ dependent variable is positive [see e.g. Kilian, 2013]. Under these assumptions, the

\footnotetext{
${ }^{21}$ Specifically, each of the $g_{i}$ corresponds to the density function of a random variable $X_{i}$ equal to $\omega_{i} W_{i, 1}+(1-$ $\left.\omega_{i}\right) W_{i, 2}$ where $\omega_{i}$ is a Bernoulli-distributed random variable of parameter $p_{i}$ and where $W_{i, 1} \sim \mathscr{N}\left(\mu_{i, 1}, \sigma_{i, 1}^{2}\right)$ and $W_{i, 2} \sim \mathscr{N}\left(\mu_{i, 2}, \sigma_{i, 2}^{2}\right)$. Imposing that the expectation and variance of $X_{i}$ are respectively equal to zero and one, these distributions depends on three parameters. We use $p_{1}=p_{2}=p_{3}=0.5, \mu_{1,1}=\mu_{2,1}=\mu_{3,1}=0.1, \sigma_{1,1}=0.5$, $\sigma_{2,1}=0.7, \sigma_{3,1}=1.3$ (which implies $\mu_{1,2}=\mu_{2,2}=\mu_{3,2}=-0.1, \sigma_{1,2}=1.32, \sigma_{2,2}=1.22$ and $\sigma_{3,2}=0.54$ ).

${ }^{22} \mathrm{We}$ associate a decrease in economic activity with an increase in the unemployment rate.
} 
structural shocks are given by $S^{-1} u_{t}$. It is easily seen that the ICA approach provides the same structural shocks as in the previous recursive approach, up to permutations and sign changes, if $C \in \mathscr{P}(I d)$, where $\mathscr{P}(I d)$ is the set of matrices obtained by permutation and sign change of the columns of the identity matrix. ${ }^{23}$ It is important to stress that, contrary to the ICA, the recursive approach assumes, potentially wrongly, that the contemporaneous impacts of some structural shocks on given variables are null and that this kind of assumption can be tested. Using the second method described in Section 2.5, we have tested two different sets of such SRRs, which correspond to two different ordering of the endogenous variables, as will be explained below. The null hypothesis of these tests is $H_{0}=(C \in \mathscr{P}(I d)) .{ }^{24}$

Typical SRRs state that monetary policy shocks have neither a contemporaneous effect on economic activity, nor on inflation [see e.g. Bernanke and Blinder (1989), Christiano, Eichenbaum and Evans (2005) or Boivin and Giannoni, 2009]. Additional SRRs are used to disentangle the remaining two shocks. A possibility is to impose that inflation is contemporaneously impacted by only one structural shock, while economic activity is affected by two of them. In this context, the test of the null hypothesis has to be performed with the macroeconomic variables ordered as follows: $Y_{t}=\left[\pi_{t}, y_{t}, r_{t}\right]$ (SRR Scheme 1, say). Indeed, in this case, the impact of the third shock $\varepsilon_{3, t}$ on $Y_{t}$ is of the form $\left[0,0, s_{3,3}\right]^{\prime}$, where we denote by $s_{i, j}$ the element $(i, j)$ of matrix $S$. Therefore, this structural shock satisfies the restrictions put on the monetary policy shock. Further, the instantaneous impacts of the first and the second components of $\varepsilon_{t}$ are respectively $\left[s_{1,1}, s_{2,1}, s_{3,1}\right]^{\prime}$ and $\left[0, s_{2,2}, s_{3,2}\right]^{\prime}$. Hence, inflation is instantaneously affected by a single shock $\left(\varepsilon_{1, t}\right)$ as requested. Alternatively, if economic activity is contemporaneously affected by a single shock, then the null hypothesis will be tested on the macrovariables with the new ordering $Y_{t}=\left[y_{t}, \pi_{t}, r_{t}\right]^{\prime}$ (SRR Scheme 2). Remark that the IRFs of the identified monetary policy shocks resulting from these two SRR schemes are identical. ${ }^{25}$

\footnotetext{
${ }^{23} \mathscr{P}(I d)$ contains $2^{n} n$ ! different matrices, that is 48 matrices for $n=3$.

${ }^{24}$ The two sets of SRRs that we consider result in two different sets of estimated structural shocks. By contrast, changing the ordering of the endogenous variables affects the ICA-based estimate of $C$, but not the associated structural shocks. Let us denote by $S_{i}$ the Cholesky decomposition (with positive diagonal entries) of $\Sigma_{i}$, where $\Sigma_{i}$ is the covariance matrix of the residuals obtained for the $i^{t h}(i \in\{1,2\})$ ordering of the endogenous variables (this ordering being consistent with the $i^{t h}$ set of SRRs). Let us further denote by $P$ the permutation matrix that is such that $u_{t}^{(1)}=P u_{t}^{(2)}$, where $u_{t}^{(i)}$ is the vector of residuals resulting from the $i^{t h}$ ordering. Then we have $C_{1}=S_{1}^{-1} P S_{2} C_{2}$, where $C_{i}$ is the estimate of $C$ associated with the $i^{\text {th }}$ ordering of the dependent variables.

${ }^{25}$ Let us denote by $\Sigma_{1}$ and $\Sigma_{2}$ the covariance matrices of the VAR residuals obtained under SRR Scheme 1 and SRR Scheme 2, respectively. (We have $\Sigma_{2}=P \Sigma_{1} P^{\prime}$ where $P$ is a permutation matrix that permutes the first two elements of a three-dimensional vector.) Under SRR Scheme 1 (respectively Scheme 2), the instantaneous impact of the identified monetary policy shock on $Y_{t}$ corresponds to the last column of $S_{1}$ (resp. $S_{2}$ ), which is the matrix resulting from the Cholesky decomposition of $\Sigma_{1}$ (resp. $\Sigma_{2}$ ) whose diagonal elements are positive. For SRR scheme $i$, this instantaneous impact is $\left[0,0, s_{3,3}^{(i)}\right]^{\prime}$, where $s_{3,3}^{(i)}$ is the $(3,3)$ element of $S_{i}$. Further, we have $s_{3,3}^{(1)}=s_{3,3}^{(2)}$. Indeed, the $j^{\text {th }}$ diagonal element of $S_{i}$ corresponds to the standard deviation of the residuals of the
} 
The bottom of Table 4 reports the $p$-values obtained for each scheme and each VAR model. The SRR schemes are rejected at the 5\% significance level for the VAR models featuring the output gap as a proxy for economic activity. The $p$-values are higher when the unemployment gap is used and, in that case, the SRR schemes cannot be rejected at the $10 \%$ significance level.

Figure 2 displays the impulse response functions resulting from the ICA approach (black solid lines) and compare them to those based on the two considered SRR Schemes (black dashed lines and grey solid lines). The responses to the monetary-policy shock and to the demand shocks are relatively close for the different methods. The difference is more marked for the supply shock, where the impact on economic activity is stronger in the ICA case. Consistently with the results of the test detailed above, there are less graphical differences between the ICAbased and the SRR-based IRFs when the unemployment gap is used to measure the economic activity.

\section{Concluding Remarks}

There is a huge literature proposing semi-parametric estimation methods for the mixing matrix in models with independent components. These methods notably include pseudo maximum likelihood approaches. The standard literature focuses on the numerical properties of these methods such as their numerical convergence, but generally neglects their statistical properties such as the statistical convergence and asymptotic distribution. The aim of our paper was to consider these statistical properties. In particular:

i) we show that the one-unit PML approaches, often used in practice, are not statistically consistent;

ii) we derive the necessary and sufficient identification conditions for multi-unit PML and recursive PML approaches, whereas only sufficient conditions have been derived in the literature;

iii) we show that the multi-unit PML approaches under the constraint of orthogonal mixing matrix are consistent and we provide the asymptotic distribution of the multi-unit PML estimator;

iv) we show - and exploit on real data - the identifiability of the structural shocks and of the impulse response functions in VAR models with non-Gaussian errors;

regression of $u_{j, t}$ on $u_{1, t}, \ldots, u_{j-1, t}$ (this relates to the Gram-Schmidt orthogonalisation procedure); therefore, $s_{n, n}^{(1)}$ does not depend on the order of the first $n-1$ elements of $u_{t}$. The IRFs of the monetary shocks resulting from both SRR schemes are therefore the same because the initial shocks as well as the following dynamics (captured by the VAR autoregressive matrices) are the same. 
v) we show that the usual identification restrictions, such as short-run restrictions, are in fact over-identification restrictions and that these restrictions can be tested.

PML approaches are largely used in practice even if they do not allow to reach the (semi-) parametric efficiency bound. Semi-parametric efficient methods have been introduced in the more theoretical literature. These methods are however more difficult to implement than the PML approaches. There is a clear trade-off between statistical efficiency and numerical simplicity [see the comparison of performances in Figure 1 of Chen, Bickel (2005)]. Moreover, they are often difficult to extend to a dynamic framework, especially to the consistent estimation of the moving average parameters $C_{j}, j=-\infty, \ldots,+\infty$, from observations of a stationary process satisfying:

$$
Y_{t}=\sum_{j=-\infty}^{\infty} C_{j} \varepsilon_{t-j}
$$

[see e.g. Gourieroux, Monfort (2014), Gourieroux, Jasiak (2015), for the estimation of such parameters by covariance estimators]. 


\section{R E F E R E N C E S}

Amari, S., and J., Cardoso (1997): "Blind Source Separation. Semi-Parametric Structural Approach", IEEE Trans. on Signal Processing, 45, 2692-2700.

Anderson, T. (1984): "An Introduction to Multivariate Statistical Analysis", New-York, Wiley.

Baten, W. D. (1934): "The Probability Law for the Sum of n Independent Variables, Each Subject to the Law $(2 h)-1 \operatorname{sech}(\pi x / 2 h)$ ", Bulletin of the American Mathematical Society, 40, 284-290.

Bell, A., and T., Sejnowski (1995): "An Information-Maximization Approach to Blind Separation and Blind Deconvolution", Neural Computation, 7, 1129-1159.

Belouchrani, A., Abed-Meraim, K., Cardoso, J.F., and E., Moulines (1997): "A Blind Source Separation Technique Using Second-Order Statistics", IEEE Trans. On Signal Processing, 45, 434-444.

Bernanke, B. (1986): "Alternative Explanations of the Money-Income Correlation", vol 25 of Carnegie-Rochester Conference Series on Public Policy, 49-99, North-Holland.

Bernanke, B. and A., Blinder (1992): "The Federal Funds Rate and the channels of monetary transmission". American Economic Review, 82(4), 901-21.

Blanchard, O., and D., Quah (1989): "The Dynamic Effects of Aggregate Demand and Supply Disturbances", American Economic Review, 79, 655-673.

Boivin, J., and M.P., Giannoni (2006): "Has Monetary Policy Become More Effective?", The Review of Economics and Statistics, 88(3), 445-462.

Bonhomme, S., and J.M., Robin (2009): "Consistent Noisy Independent Component Analysis", Journal of Econometrics, 149, 12-25.

Canova, F. (1995): "Vector autoregressive models: specifications, estimation, inference, and forecasting", in Handbook of Applied Econometrics. Macroeconomics, ed. by M. Pesaran, and M. Wickens. Blackwell, Oxford UK and Cambridge USA.

Cardoso, J. (1999): "High-order Contrasts for Independent Component Analysis", Neural Comput., 11, 157-192.

Cardoso, J., and B.H., Laheld (1996): "Equivariant Adaptive Source Separation", IEEE Tran. on Signal Processing, 44, 3017-3030.

Cardoso, J., and A., Souloumiac (1993): "Blind Beamforming for Non Gaussian Signals", IEE. Proceedings, F, 140, 362-370.

Chari, V., Kehoe, P., and E., McGrattan (2008): "Are Structural VARs with Long Run 
Restrictions Useful in Developing Business Cycle Theory ?", Journal of Monetary Economics, 55, 1337-1 352 .

Chen, A., and P., Bickel (2005): "Consistent Independent Component Analysis and Prewhitening", IEEE Transactions on Signal Processing, 53, 3625-3632.

Chen, B., Choi, J., and J.C., Escanciano (2012): "Testing for Fundamental Moving Average Representation", DP Indiana University.

Christiano, L.J., Eichenbaum, M., and C.L. Evans (2001): "Nominal Rigidities and the Dynamic Effects of a Shock to Monetary Policy", Journal of Political Economy, 113(1), 1-45.

Christiano, L., Eichenbaum, M., and R., Vigfusson (2006): "Alternative Procedures for Estimating Vector Autoregressions Identified with Long Run Restrictions", Journal of the European Economic Association", vol 4, 475-483.

Comon, P. (1994): "Independent Component Analysis: A New Concept ?", Signal Processing, 36, 287-314.

Comon, P., Jutten, C., and J., Herault (1991): "Blind Separation of Sources, Part II: Problems Statement", Signal Processing, 24, 11-20.

Degerine, S., and R., Malki (2000): "Second-Order Blind Separation of Sources Based on Canonical Partial Innovations", IEEE Trans. on Signal Processing, 48, 629-641.

Erceg, C., Guerrieri, L., and C., Gust (2005): "Can Long Run Restriction Identify Technology Shocks ?", Journal of the European Economic Association, 3, 1237-1278.

Eriksson, J., and V., Koivunen (2004): "Identifiability, Separability and Uniqueness of Linear ICA Models", IEEE Signal Processing Letters, 11, 601-604.

Faust, J., and E., Leeper (1997): "When Do Long Run Identifying Restrictions Give Reliable Results ?", Journal of Business and Economic Statistics, 15, 345-353.

Gourieroux, C., and J., Jasiak (2015): "Semi-Parametric Estimation of Noncausal Vector Autoregression", CREST DP.

Gourieroux, C., and A., Monfort (1995): "Statistics and Econometric Models", Cambridge University Press.

Gourieroux, C., and A., Monfort (2014): "Revisiting Identification and Estimation in Structural VARMA Models", CREST DP.

Hastie, T., and R., Tibshirani (2002): "Independent Component Analysis Through Product Density Estimators", DP Stanford University.

Hyvarinen, A. (1997): "Independent Component Analysis by Minimization of Mutual Information", Helsinki University of Technology.

Hyvarinen, A. (1999): "Fast and Robust Fixed-Point Algorithms for Independent Component Analysis", IEEE Transactions on Neural Networks, 10, 626-634. 
Hyvarinen, A., Karhunen, J., and E., Oja (2001): "Independent Component Analysis", Wiley.

Hyvarinen, A., and E., Oja (1997): "A Fast Fixed Point Algorithm for Independent Component Analysis", Neural Computation, 9, 1483-1492.

Hyvarinen, A., and E., Oja (2000): "Independent Component Analysis: Algorithms and Applications", Neural Networks, 13, 411-430.

Ilmonen, P. (2013): "On Asymptotic Properties of the Scatter Matrix Based Estimates for Complex Valued Independent Component Analysis", Probability Letters, 83, 1219-1226.

Ilmonen, P., Nordhausen, K., Oja, H., and E., Ollila (2012): "On Asymptotics of ICA Estimators and Their Performance Indices", DP.

Jennrich, R (1969): "Asymptotic Properties of Nonlinear Least Squares Estimators", The Annals of Mathematical Statistics, 40, 633-643.

Jutten, C., and J., Herault (1991): "Blind Separation of Sources. Part 1: An Adaptive Algorithm Based on Neuromimetic Structure", Signal Processing, 24, 1-10.

Kaiser, H. (1958): "The Varimax Criterion for Analytic Rotation in Factor Analysis", Psychometrika, 23, 187-200.

Kilian, L. (2013): "Structural Vector Autoregressions", Chapters, in: Handbook of Research Methods and Applications in Empirical Macroeconomics, Chapter 22, 515-554, Edward Elgar.

Lawley, D., and A., Maxwell (1971): "Factor Analysis in a Statistical Method", Butterworth, London.

Leeper, E.M., Sims, C.A. and T., Zha (1996): "What Does Monetary Policy do?", Brookings Papers on Economic Activity, 2, 1-78.

Lütkepohl, H. (2005): "New Introduction to Multiple Time Series Analysis", SpringerVerlag, Berlin.

Miettinen, J., Nordhausen, K., Oja, H., and S., Taskinen (2014): "Deflation-Based Fast ICA with Adaptive Choices of Nonlinearities", IEEE Transactions on Signal Processing, 1-9.

Moneta, A., Entner, D., Hoyer, P., and A., Coad (2013): "Causal Inference by Independent Component Analysis: Theory and Applications", Oxford Bulletin of Economics and Statistics, 75, 705-730.

Mountford, A. and H., Uhlig (2009): "What Are the Effects of Fiscal Policy Shocks ?", Journal of Applied Econometrics, 24, 960-992.

Ollila, E. (2010): "The Deflation-Based FastICA Estimator: Statistical Analysis Revisited", IEEE Transaction in Signal Processing, 58, 175-189.

Pham, D., and P., Garat (1997): "Blind Separation of Mixture of Independent Sources Through a Quasi-Maximum Likelihood Approach", IEEE Transactions on Signal Processing, 
$45,1712-1725$.

Reyhani, N., Ylipaavalniemi, J., Vigario, R., and O., Erkki (2012): "Consistency and Asymptotic Normality of FastICA and Bootstrap FastICA", Signal Processing, 92, 1767-1778.

Rubio-Ramirez, J., Waggoner, D., and T., Zha (2010) "Structural Vector Autoregression: Theory of Identification and Algorithms for Inference", Review of Economic Studies, 77, 665696.

Sims, C. A. (1980a): "Macroeconomics and Reality", Econometrica, 48(1), 1-48.

Sims, C. A. (1980b): "Comparison of Interwar and Postwar Business Cyles: Monetarism Re-considered,? American Economic Review, 70, 250-257.

Sims, C. A. (1986): "Are Forecasting Models Usable for Policy Analysis?", Federal Reserve Bank of Minneapolis Quarterly Review, 10, 1-16.

Sims, C. A. (1992): "Interpreting the Macroeconomic Time Series Facts: The Effects of Monetary Policy", European Economic Review, 36(5), 975-1011.

Stock, J.H. and M.W., Watson (2001): "Vector Autoregressions", The Journal of Economic Perspectives, 15(4), 101-115.

Tong, L., Soon, V., Huang, Y., and R., Liu (1990): "Amuse: A New Blind Identification Algorithm", in Proc. IEEE ISCAS, 1784-1787, New-Orleans, May.

Tong, L., Soon, V., Huang, Y., and R., Liu (1991): "Indeterminacy and Identifiability of Blind Identification", IEEE Trans. Signal Processing, 38, 499-509.

Uhlig, H. (2005): "What Are the Effects of Monetary Policy on Output? Result From an Agnostic Identification Procedure", Journal of Monetary Economics, 52, 381-419.

Vlassis, N., and Y., Motomura (2001): "Efficient Source Adaptivity in Independent Component Analysis", IEEE Trans. Neural Networks, 12, 559-565.

Watson, M.W. (1994): "Vector Autoregressions and Cointegration", Handbook of Econometrics, Vol. 4, R.F. Engle and D. McFadden (eds), Amsterdam: Elsevier Science Ltd., 28442915.

Wei, T. (2014): "The Convergence and Asymptotic Analysis of the Generalized Symmetric Fast ICA Algorithm", DP University of Lille. 


\section{Appendix 1}

\section{Expansion of the Cayley's Representation of an Orthogonal Matrix}

Let us consider the second-order expansion of $C(A)=(I d+A)(I d-A)^{-1}$ with respect to $A$. Denoting $A=A_{0}+\Delta A$, where $\Delta A$ is a small skew-symmetric matrix, we have:

$$
\begin{aligned}
C(A) & =\left(I d+A_{0}+\Delta A\right)\left(I d-A_{0}-\Delta A\right)^{-1} \\
& =\left(I d+A_{0}+\Delta A\right)\left\{\left[I d-\Delta A\left(I d-A_{0}\right)^{-1}\right]\left(I d-A_{0}\right)\right\}^{-1} \\
& =\left(I d+A_{0}+\Delta A\right)\left(I d-A_{0}\right)^{-1}\left[I d-\Delta A\left(I d-A_{0}\right)^{-1}\right]^{-1} \\
& =\left[C\left(A_{0}\right)+\Delta A\left(I d-A_{0}\right)^{-1}\right]\left[I d+\Delta A\left(I d-A_{0}\right)^{-1}+\Delta A\left(I d-A_{0}\right)^{-1} \Delta A\left(I d-A_{0}\right)^{-1}\right] \\
& +o\|\Delta A\|^{2},
\end{aligned}
$$

where ||$.||$ denotes any matrix norm. Hence, we have:

$$
\begin{aligned}
C(A) & =C\left(A_{0}\right)+\left\{\Delta A\left(I d-A_{0}\right)^{-1}+C\left(A_{0}\right) \Delta A\left(I d-A_{0}\right)^{-1}\right\} \\
& +\Delta A\left(I d-A_{0}\right)^{-1} \Delta A\left(I d-A_{0}\right)^{-1}+C\left(A_{0}\right) \Delta A\left(I d-A_{0}\right)^{-1} \Delta A\left(I d-A_{0}\right)^{-1}+o\|\Delta A\|^{2} \\
& =C\left(A_{0}\right)+\left[I d+C\left(A_{0}\right)\right] \Delta A\left(I d-A_{0}\right)^{-1} \\
& +\left[I d+C\left(A_{0}\right)\right] \Delta A\left(I d-A_{0}\right)^{-1} \Delta A\left(I d-A_{0}\right)^{-1}+o(\|\Delta A\|)^{2} .
\end{aligned}
$$

Since:

$$
I d+C\left(A_{o}\right)=I d+\left(I d+A_{0}\right)\left(I d-A_{0}\right)^{-1}=2\left(I d-A_{0}\right)^{-1}
$$

we also get:

$$
\begin{array}{r}
C(A)=C\left(A_{0}\right)+2\left(I d-A_{0}\right)^{-1} \Delta A\left(I d-A_{0}\right)^{-1} \\
+2\left(I d-A_{0}\right)^{-1} \Delta A\left(I d-A_{0}\right)^{-1} \Delta A\left(I d-A_{0}\right)^{-1}+o(\|\Delta A\|)^{2} .
\end{array}
$$

By using the equalities $\Delta A^{\prime}=-\Delta A$, and $A_{0}^{\prime}=-A_{0}$, we deduce the expansion of the transpose $C^{\prime}(A)$ :

$$
\begin{array}{r}
C^{\prime}(A)=C^{\prime}\left(A_{0}\right)-2\left(I d+A_{0}\right)^{-1} \Delta A\left(I d+A_{0}\right)^{-1} \\
+2\left(I d+A_{0}\right)^{-1} \Delta A\left(I d+A_{0}\right)^{-1} \Delta A\left(I d+A_{0}\right)^{-1}+o(\|\Delta A\|)^{2}, \\
\text { with } C^{\prime}(A)=(I d+A)^{-1}(I d-A) .
\end{array}
$$

We also deduce:

$$
\begin{aligned}
C^{\prime}(A) Y_{t} & =C^{\prime}(A) C\left(A_{0}\right) \varepsilon_{t} \\
& =\varepsilon_{t}-2\left(I d+A_{0}\right)^{-1} \Delta A\left(I d+A_{0}\right)^{-1} C\left(A_{0}\right) \varepsilon_{t} \\
& +2\left(I d+A_{0}\right)^{-1} \Delta A\left(I d+A_{0}\right)^{-1} \Delta A\left(I d+A_{0}\right)^{-1} C\left(A_{0}\right) \varepsilon_{t}+o(\|\Delta A\|)^{2} \\
& =\varepsilon_{t}-2\left(I d+A_{0}\right)^{-1} \Delta A\left(I d-A_{0}\right)^{-1} \varepsilon_{t} \\
& +2\left(I d+A_{0}\right)^{-1} \Delta A\left(I d+A_{0}\right)^{-1} \Delta A\left(I d-A_{0}\right)^{-1} \varepsilon_{t}+o(\|\Delta A\|)^{2}
\end{aligned}
$$




\section{Appendix 2}

\section{Local Concavity of the asymptotic Pseudo Log-Likelihood Functions}

\section{A.2.1 PML estimator (with SIR3)}

i) Let us first explicit the second-order expansion of the asymptotic objective function without taking into account the constraints of orthogonal $C$ matrix. We introduce the notation $c_{i}=c_{i, 0}+\delta_{i}$ where $\delta_{i}$ is small and where $c_{i, 0}$ is the $i^{t h}$ column of any matrix of $\mathscr{P}\left(C_{0}\right)$, denoted $C_{0}$ for the sake of notational simplicity. We get:

$$
\begin{aligned}
L_{\infty}(\boldsymbol{\delta}) & =E_{0}\left[\sum_{i=1}^{n} \log g_{i}\left(c_{i}^{\prime} Y_{t}\right)\right] \\
& \simeq E_{0}\left\{\sum_{i=1}^{n} \log g_{i}\left(c_{i, 0}^{\prime} Y_{t}\right)+\frac{d \log g_{i}}{d \varepsilon}\left(c_{i, 0}^{\prime} Y_{t}\right) \delta_{i}^{\prime} Y_{t}+\frac{1}{2} \frac{d^{2} \log g_{i}}{d \varepsilon^{2}}\left(c_{i, 0}^{\prime} Y_{t}\right)\left(\delta_{i}^{\prime} Y_{t}\right)^{2}\right\} .
\end{aligned}
$$

Since $Y_{t}=\sum_{j=1}^{n} c_{j, 0} \varepsilon_{j, t}$, we deduce:

$$
\begin{aligned}
L_{\infty}(\delta) & \simeq E_{0}\left[\sum_{i=1}^{n} \log g_{i}\left(\varepsilon_{i, t}\right)\right]+\sum_{i=1}^{n} \sum_{j=1}^{n} E_{0}\left[\frac{d \log g_{i}\left(\varepsilon_{i, t}\right)}{d \varepsilon} \varepsilon_{j, t}\right] \delta_{i}^{\prime} c_{j, 0} \\
& +\frac{1}{2} \sum_{i=1}^{n} \sum_{j=1}^{n} \sum_{k=1}^{n} E_{0}\left[\frac{d^{2} \log g_{i}\left(\varepsilon_{i, t}\right)}{d \varepsilon^{2}} \varepsilon_{j, t} \varepsilon_{k, t}\right] \delta_{i}^{\prime} c_{j, 0} \delta_{i}^{\prime} c_{k, 0} \\
& =E_{0}\left[\sum_{i=1}^{n} \log g_{i}\left(\varepsilon_{i, t}\right)\right]+\sum_{i=1}^{n} E_{0}\left[\frac{d \log g_{i}\left(\varepsilon_{i, t}\right)}{d \varepsilon} \varepsilon_{i, t}\right] \delta_{i}^{\prime} c_{i, 0} \\
& +\frac{1}{2} \sum_{i=1}^{n} \sum_{j=1}^{n} E_{0}\left[\frac{d^{2} \log g_{i}\left(\varepsilon_{i, t}\right)}{d \varepsilon^{2}} \varepsilon_{j, t}^{2}\right]\left(\delta_{i}^{\prime} c_{j, 0}\right)^{2},
\end{aligned}
$$

by using the independence property.

Since:

$$
E_{0}\left[\frac{d^{2} \log g_{i}\left(\varepsilon_{i, t}\right)}{d \varepsilon^{2}} \varepsilon_{j, t}^{2}\right]=E_{0}\left[\frac{d^{2} \log g_{i}\left(\varepsilon_{i, t}\right)}{d \varepsilon^{2}}\right] E_{0}\left(\varepsilon_{j, t}^{2}\right)=E_{0}\left[\frac{d^{2} \log g_{i}\left(\varepsilon_{i, t}\right)}{d \varepsilon^{2}}\right], \text { if } i \neq j,
$$

we get: 


$$
\begin{aligned}
L_{\infty}(\boldsymbol{\delta}) & \simeq E_{0}\left[\sum_{i=1}^{n} \log g_{i}\left(\varepsilon_{i, t}\right)\right]+\sum_{i=1}^{n} E_{0}\left[\frac{d \log g_{i}\left(\varepsilon_{i, t}\right)}{d \varepsilon} \varepsilon_{i, t}\right] \delta_{i}^{\prime} c_{i, 0} \\
& +\frac{1}{2} \sum_{i=1}^{n} E_{0}\left[\frac{d^{2} \log g_{i}\left(\varepsilon_{i, t}\right)}{d \varepsilon^{2}} \varepsilon_{i t}^{2}\right]\left(\delta_{i}^{\prime} c_{i, 0}\right)^{2} \\
& +\frac{1}{2} \sum_{i=1}^{n} E_{0}\left[\frac{d^{2} \log g_{i}\left(\varepsilon_{i, t}\right)}{d \varepsilon^{2}}\right]\left[\delta_{i}^{\prime} \delta_{i}-\left(\delta_{i}^{\prime} c_{i, 0}\right)^{2}\right]
\end{aligned}
$$

since $\sum_{j=1}^{n}\left(\delta_{i}^{\prime} c_{j, 0}\right)^{2}=\sum_{j=1}^{n}\left(\delta_{i}^{\prime} c_{j, 0} c_{j, 0}^{\prime} \delta_{i}\right)=\delta_{i}^{\prime} C_{0} C_{0}^{\prime} \delta_{i}=\delta_{i}^{\prime} \delta_{i}$.

This expansion of the objective function involves the $n^{2}$ infinitesimal coordinates $\Delta_{i, j} \equiv$ $-c_{i, 0}^{\prime} \delta_{j}, i, j=1, \ldots, n$, which are submitted to the $n(n+1) / 2$, restrictions of orthogonal $C$ matrix.

ii) Let us now expand the orthogonality restrictions of matrix $C$. They are equivalent to:

$$
\delta_{j}^{\prime} c_{i, 0}+\delta_{i}^{\prime} c_{j, 0}+\delta_{i}^{\prime} \delta_{j}=0, \quad i \leq j
$$

These equations show that $\delta_{i}^{\prime} c_{i, 0}=-\frac{1}{2} \delta_{i}^{\prime} \delta_{i}$ and $\delta_{j}^{\prime} c_{i, 0}+\delta_{i}^{\prime} c_{j, 0}=-\delta_{i}^{\prime} \delta_{j}$ are of second-order. Eliminating the negligible terms in the expansion of $L_{\infty}(\delta)$ and using the fact that:

$$
\delta_{i}^{\prime} \delta_{i}=\sum_{j=i}^{n}\left(\delta_{i}^{\prime} c_{j, 0}\right)^{2} \simeq \sum_{j \neq i}^{n}\left(\delta_{i}^{\prime} c_{j, 0}\right)^{2} \quad\left(\text { since }\left(\delta_{i}^{\prime} c_{i, 0}\right)^{2} \text { is negligible }\right)
$$

we get:

$$
\begin{aligned}
L_{\infty}(\boldsymbol{\delta}) & \simeq E_{0}\left[\sum_{i=1}^{n} \log g_{i}\left(\varepsilon_{i, t}\right)\right]-\frac{1}{2} \sum_{i=1}^{n} E_{0}\left[\frac{d \log g_{i}\left(\varepsilon_{i, t}\right)}{d \varepsilon} \varepsilon_{i, t}\right] \delta_{i}^{\prime} \delta_{i} \\
& +\frac{1}{2} \sum_{i=1}^{n} E_{0}\left[\frac{d^{2} \log g_{i}\left(\varepsilon_{i, t}\right)}{d \varepsilon^{2}} \varepsilon_{i, t}^{2}\right]\left(\delta_{i}^{\prime} c_{i, 0}\right)^{2}+\frac{1}{2} \sum_{i=1}^{n} E_{0}\left[\frac{d^{2} \log g_{i}\left(\varepsilon_{i, t}\right)}{d \varepsilon^{2}}\right]\left[\delta_{i}^{\prime} \delta_{i}-\left(\delta_{i}^{\prime} c_{i, 0}\right)^{2}\right] \\
& \simeq E_{0}\left[\sum_{i=1}^{n} \log g_{i}\left(\varepsilon_{i, t}\right)\right]+\frac{1}{2} \sum_{i=1}^{n} \sum_{j \neq i}\left\{E_{0}\left[\frac{d^{2} \log g_{i}\left(\varepsilon_{i, t}\right)}{d \varepsilon^{2}}-\frac{d \log g_{i}\left(\varepsilon_{i, t}\right)}{d \varepsilon} \varepsilon_{i, t}\right]\left(\delta_{i}^{\prime} c_{j, 0}\right)^{2}\right. \\
& \simeq E_{0}\left[\sum_{i=1}^{n} \log g_{i}\left(\varepsilon_{i, t}\right)\right] \\
& +\frac{1}{2} \sum_{i=1}^{n} \sum_{j>i} E_{0}\left[\frac{d^{2} \log g_{i}\left(\varepsilon_{i, t}\right)}{d \varepsilon^{2}}+\frac{d^{2} \log g_{j}\left(\varepsilon_{j, t}\right)}{d \varepsilon^{2}}-\frac{d \log g_{i}\left(\varepsilon_{i, t}\right)}{d \varepsilon} \varepsilon_{i, t}-\frac{d \log g_{j}\left(\varepsilon_{j, t}\right.}{d \varepsilon} \varepsilon_{j, t}\right]\left(\delta_{i}^{\prime} c_{j, 0}\right)^{2}
\end{aligned}
$$

since $\delta_{i}^{\prime} c_{j, 0} \simeq-\delta_{j}^{\prime} c_{i, 0}$. 
This expansion involves the $n(n-1) / 2$ functionally independent components of $\Delta=\left(\Delta_{i j}\right)$ at order 1 . The condition for local concavity follows.

\section{A.2.2 Recursive PML estimator (under SIR3)}

Let us now consider the conditions for the recursive PML estimator. At iteration $i$, the expansion of the asymptotic objective function becomes:

$$
\begin{aligned}
L_{\infty}\left(\delta_{i}\right) & \simeq E_{0} \log g_{i}\left(\varepsilon_{i, t}\right)+E_{0}\left(\frac{d \log g_{i}\left(\varepsilon_{i, t}\right)}{d \varepsilon} \varepsilon_{i, t}\right) \delta_{i}^{\prime} c_{i, 0} \\
& +\frac{1}{2} \sum_{j=1}^{n} E_{0}\left[\frac{d^{2} \log g_{i}\left(\varepsilon_{i, t}\right)}{d \varepsilon^{2}} \varepsilon_{j, t}^{2}\right]\left(\delta_{i}^{\prime} c_{j, 0}\right)^{2} .
\end{aligned}
$$

The restrictions for orthogonal matrix $C$ are:

$$
\begin{aligned}
c_{i}^{\prime} c_{j, 0} & =0, j<i, \\
c_{i}^{\prime} c_{i} & =1,
\end{aligned}
$$

and are equivalent to:

$$
\delta_{i}^{\prime} c_{j, 0}=0, \forall j<i, 2 \delta_{i}^{\prime} c_{i, 0}+\delta_{i}^{\prime} \delta_{i}=0
$$

Since $\delta_{i}^{\prime} c_{i, 0}=-(1 / 2) \delta_{i}^{\prime} \delta_{i}$ is of order 2 , we have:

$$
\delta_{i}^{\prime} \delta_{i}=\sum_{j=1}^{n}\left(\delta_{i} c_{j, 0}\right)^{2} \simeq \sum_{j>i}\left(\delta_{i} c_{j, 0}\right)^{2}
$$

and the expansion of the objective function becomes:

$$
L_{\infty}\left(\delta_{i}\right) \simeq E_{0} \log g_{i}\left(\varepsilon_{i, t}\right)+\frac{1}{2}\left\{E_{0}\left[\frac{d^{2} \log g_{i}\left(\varepsilon_{i, t}\right)}{d \varepsilon^{2}}\right]-E_{0}\left[\frac{d \log g_{i}\left(\varepsilon_{i, t}\right)}{d \varepsilon} \varepsilon_{i, t}\right]\right\} \delta_{i}^{\prime} \delta_{i}
$$

Thus the condition for local concavity is :

$$
E_{0}\left[\frac{d^{2} \log g_{i}\left(\varepsilon_{i, t}\right)}{d \varepsilon^{2}}\right]-E_{0}\left[\frac{d \log g_{i}\left(\varepsilon_{i, t}\right)}{d \varepsilon} \varepsilon_{i, t}\right]<0,
$$

and has to be written for $i=1, \ldots, n-1$. 


\section{Appendix 3}

\section{Consistency of the PML with Auxiliary Parameters and Recursive PML Estimator.}

\section{A.3.1 PML with auxiliary parameters}

The asymptotic FOC for optimization problem (3.5) are:

$$
\left\{\begin{array}{l}
E_{0}\left[\frac{d \log g_{i}}{d \varepsilon}\left(\frac{c_{i}^{\prime} Y_{t}}{\sigma_{i}}\right) \frac{Y_{t}}{\sigma_{i}}\right]-\lambda_{i, i} c_{i}=0 \\
E_{0}\left[\frac{d \log g_{i}}{d \varepsilon}\left(\frac{c_{i}^{\prime} Y_{t}}{\sigma_{i}}\right) \frac{c_{i}^{\prime} Y_{t}}{\sigma_{i}^{2}}+\frac{1}{\sigma_{i}}\right]=0
\end{array}\right.
$$

They are equivalent to:

$$
\left\{\begin{array}{l}
E_{0}\left[\frac{d \log g_{i}}{d \varepsilon}\left(\frac{c_{i}^{\prime} Y_{t}}{\sigma_{i}}\right) \frac{c_{j, 0}^{\prime} Y_{t}}{\sigma_{i}}\right]-\lambda_{i, i} c_{j, 0}^{\prime} c_{i}=0, \quad \forall j \neq i, \\
E_{0}\left[\frac{d \log g_{i}}{d \varepsilon}\left(\frac{c_{i}^{\prime} Y_{t}}{\sigma_{i}}\right) \frac{c_{i, 0}^{\prime} Y_{t}}{\sigma_{i}}\right]-\lambda_{i, i} c_{i, 0}^{\prime} c_{i}=0, \\
E_{0}\left[\frac{d \log g_{i}}{d \varepsilon}\left(\frac{c_{i}^{\prime} Y_{t}}{\sigma_{i}}\right) \frac{c_{i}^{\prime} Y_{t}}{\sigma_{i}^{2}}+\frac{1}{\sigma_{i}}\right]=0 .
\end{array}\right.
$$

The first subsystem is satisfied for $c_{i}=c_{i, 0}$ and any value of $\sigma_{i}$. Then the third subsystem is used to find the appropriate value of $\sigma_{i}$, which is generally different from $\sigma_{i, 0}$, whereas the second equation fixes the asymptotic value of the Lagrange multiplier.

\section{A.3.2 Jacobian adjusted PML with auxiliary parameters}

The constrained optimization problem is:

$$
\left\{\begin{array}{l}
\max _{B} \sum_{t=1}^{T}\left[\sum_{i=1}^{n}\left\{\log g_{i}\left(\frac{b_{i}^{\prime} Y_{t}}{\sigma_{i}}\right)+\log \operatorname{det} B-\frac{1}{2} \log \sigma_{i}^{2}\right],\right. \\
\text { s.t.: } b_{i}^{\prime} b_{i}=1, i=1, \ldots, n,
\end{array}\right.
$$

where $B=\left(\begin{array}{c}b_{1}^{\prime} \\ \vdots \\ b_{n}^{\prime}\end{array}\right)$ 
The associate asymptotic criterion is:

$$
\sum_{i=1}^{n} E_{0}\left[\log g_{i}\left(\frac{b_{i}^{\prime} Y_{t}}{\sigma_{i}}\right)+\log \operatorname{det} B-\frac{1}{2} \log \sigma_{i}^{2}\right],
$$

and the asymptotic FOC for $b_{i}$ are:

$$
E_{0}\left[Y_{t} \frac{d \log g_{i}}{d \varepsilon}\left(\frac{b_{i}^{\prime} Y_{t}}{\sigma_{i}}\right)\right]-\lambda_{i, i} b_{i}+b^{i}=0, i=1, \ldots, n
$$

where the derivative of $\log \operatorname{det} B$ with respect to $B$ is $\left(B^{-1}\right)^{\prime}, b^{i}$ denotes the $i^{\text {th }}$ column of $B^{-1}$, and $\lambda_{i, i} / 2$ the Lagrange multiplier corresponding to the restrictions $b_{i}^{\prime} b_{i}=1$.

Let us now check if $C_{0}^{-1}$ is solution of these asymptotic FOC. These FOC become:

$$
\sum_{j=1}^{n} c_{0, j} E_{0}\left[\varepsilon_{j, t} \frac{d \log g_{i}\left(\varepsilon_{i, t} / \sigma_{i}\right)}{d \varepsilon}\right]-\lambda_{i, i} c_{0}^{i}+c_{0, i}=0
$$

where $c_{0}^{i}$ is the $i^{\text {th }}$ row of $C_{0}^{-1}$,

$$
\text { or } c_{0, i}\left\{E_{0}\left[\varepsilon_{i, t} \frac{d \log g_{i}\left(\varepsilon_{i, t} / \sigma_{i}\right)}{d \varepsilon}\right]+1\right]-\lambda_{i, i} c_{0}^{i}=0 .
$$

Then, we have to distinguish two cases:

i) If the matrix $C_{0}$ is not orthogonal, these FOC are not satisfied.

ii) If the matrix $C_{0}$ is orthogonal, we have $c_{0}^{i}=c_{0, i}$. The constraints of the optimization problem are satisfied and the FOC above provides the value of the (asymptotic) Lagrange multiplier for a given value of $\sigma_{i}$ :

$$
\lambda_{i, i}=E_{0}\left[\varepsilon_{i, t} \frac{d \log g_{i}\left(\varepsilon_{i, t} / \sigma_{i}\right)}{d \varepsilon}\right]+1 .
$$

The value of $\sigma_{i}$ is deduced from the asymptotic FOC for $\sigma_{i}$ :

$$
E_{0}\left[-\frac{\varepsilon_{i, t}}{\sigma_{i}^{2}} \frac{d \log g_{i}\left(\varepsilon_{i, t} / \sigma_{i}\right)}{d \varepsilon}\right]-\frac{1}{\sigma_{i}}=0 .
$$

\section{A.3.3 Asymptotic FOC for the recursive PML estimator}

Let us denote by $\lambda_{i, i} / 2, \lambda_{i, j}, j<i$, the Lagrange multipliers associated with restrictions $c_{i}^{\prime} c_{i}=$ $1, c_{i}^{\prime} c_{j, 0}=0, j<i$. The derivative of the asymptotic Lagrangian associated with the optimization 
problem (3.8) provides the system:

$$
E_{0}\left[Y_{t} \frac{d \log g_{i}}{d \varepsilon}\left(c_{i}^{\prime} Y_{t}\right)\right]-\lambda_{i, i} c_{i}-\Sigma_{j<i} \lambda_{i, j} c_{j, 0}=0,
$$

with $c_{i}^{\prime} c_{i}=1, c_{i}^{\prime} c_{j, 0}=0, j<i$.

By multiplying the first equation by $c_{i}^{\prime}$, and by $c_{j, 0}^{\prime}, j=1, \ldots, i-1$, and using the orthogonality conditions, including $c_{j, 0}^{\prime} c_{k, 0}=0, k \neq j \leq i-1 c_{j, 0}^{\prime} c_{j, 0}=1, j=1, \ldots, i-1$, we get:

$$
\lambda_{i, i}=E_{0}\left[c_{i}^{\prime} Y_{t} \frac{d \log g_{i}}{d \varepsilon}\left(c_{i}^{\prime} Y_{t}\right)\right], \lambda_{i, j}=E_{0}\left[c_{j, 0}^{\prime} Y_{t} \frac{d \log g_{i}}{d \varepsilon}\left(c_{i}^{\prime} Y_{t}\right)\right]=E_{0}\left[\varepsilon_{j, t} \frac{d \log g_{i}}{d \varepsilon}\left(c_{i}^{\prime} Y_{t}\right)\right] .
$$

Thus the system becomes:

$$
\begin{aligned}
& E_{0}\left\{\frac{d \log g_{i}}{d \varepsilon}\left(c_{i}^{\prime} Y_{t}\right)\left[\sum_{j=i}^{n} c_{j, 0} \varepsilon_{j, t}-c_{i}^{\prime} Y_{t} c_{i}-\Sigma_{j<i} \varepsilon_{j, t} c_{j, 0}\right]\right\}=0, \\
& c_{i}^{\prime} c_{i}=1, c_{i}^{\prime} c_{j, 0}=0, j<i .
\end{aligned}
$$

We see that the true $c_{i, 0}$ is solution of this system. Indeed for $c_{i}=c_{i, 0}$ the first subsystem becomes:

$$
\begin{aligned}
& E_{0}\left\{\frac{d \log g_{i}\left(\varepsilon_{i, t}\right)}{d \varepsilon}\left[\sum_{j=i}^{n} c_{j, 0} \varepsilon_{j, t}-\varepsilon_{i, t} c_{i, 0}\right]\right\} \\
= & E_{0}\left\{\frac{d \log g_{i}\left(\varepsilon_{i, t}\right)}{d \varepsilon}\left[c_{i, 0} \varepsilon_{i, t}-c_{i, 0} \varepsilon_{i, t}\right]\right\}=0 .
\end{aligned}
$$

We deduce from the computation above the identification assumption A.3. 


\section{Appendix 4}

\section{Asymptotic Distribution of the PML Estimator}

Let us denote by $C_{0}$ the unique value of $C$ providing the global maximum of the asymptotic criterion $\sum_{i=1}^{n} E_{0}\left[\log g_{i}\left(c_{i}^{\prime} Y_{t}\right)\right]$ (assuming that the $g_{i}$ are different and asymmetric, as well as true distributions of the $\left.\varepsilon_{j, t}, j=1, \ldots, n\right)$.

Consider the finite sample FOC (2.9):

$$
\left\{\begin{array}{l}
\sum_{t=1}^{T} \hat{c}_{j}^{\prime} Y_{t} \frac{d \log g_{i}}{d \varepsilon}\left(\hat{c}_{i}^{\prime} Y_{t}\right)-\sum_{t=1}^{T} \hat{c}_{i}^{\prime} Y_{t} \frac{d \log g_{j}}{d \varepsilon}\left(\hat{c}_{j}^{\prime} Y_{t}\right)=0, i<j \\
\hat{c}_{i}^{\prime} \hat{c}_{j}=0, i<j, \hat{c}_{i}^{\prime} \hat{c}_{i}=1, i=1, \ldots, n
\end{array}\right.
$$

Let us denote by $\hat{\delta}_{i}=\hat{c}_{i}-c_{i, 0}$ the difference between the PML estimator and the true value. A first-order expansion of the equations in (a.1) gives:

$$
\left\{\begin{array}{l}
\sum_{t=1}^{T}\left(c_{j, 0}^{\prime}+\hat{\delta}_{j}^{\prime}\right) Y_{t} \frac{d \log g_{i}}{d \varepsilon}\left(c_{i, 0}^{\prime} Y_{t}\right)+\sum_{t=1}^{T} c_{j, 0}^{\prime} Y_{t} \frac{d^{2} \log g_{i}}{d \varepsilon^{2}}\left(c_{i, 0}^{\prime} Y_{t}\right) \hat{\delta}_{i}^{\prime} Y_{t} \\
-\sum_{t=1}^{T}\left(c_{i, 0}^{\prime}+\hat{\delta}_{i}^{\prime}\right) Y_{t} \frac{d \log g_{j}}{d \varepsilon}\left(c_{j, 0}^{\prime} Y_{t}\right)-\sum_{t=1}^{T} c_{i, 0}^{\prime} Y_{t} \frac{d^{2} \log g_{j}}{d \varepsilon^{2}}\left(c_{j, 0}^{\prime} Y_{t}\right) \hat{\delta}_{j}^{\prime} Y_{t} \simeq 0, i<j, \\
c_{i, 0}^{\prime} \hat{\boldsymbol{\delta}}_{j}+c_{j, 0}^{\prime} \hat{\boldsymbol{\delta}}_{i} \simeq 0, i<j, c_{i, 0}^{\prime} \hat{\boldsymbol{\delta}}_{i} \simeq 0, i=1, \ldots, n .
\end{array}\right.
$$

Let us focus on the first subsystem. This subsystem is equivalent to:

$$
\begin{aligned}
& \sum_{t=1}^{T}\left[\varepsilon_{j, t} \frac{d \log g_{i}\left(\varepsilon_{i, t}\right)}{d \varepsilon}-\varepsilon_{i, t} \frac{d \log g_{j}\left(\varepsilon_{j, t}\right)}{d \varepsilon}\right] \\
+ & \sum_{t=1}^{T}\left\{\left[\varepsilon_{j, t} \frac{d^{2} \log g_{i}\left(\varepsilon_{i, t}\right)}{d \varepsilon^{2}}-\frac{d \log g_{j}\left(\varepsilon_{j, t}\right)}{d \varepsilon}\right] \varepsilon_{t}^{\prime}\right\} C_{0}^{\prime} \hat{\delta}_{i} \\
- & \sum_{t=1}^{T}\left\{\left[\varepsilon_{i, t} \frac{d^{2} \log g_{j}\left(\varepsilon_{j, t}\right)}{d \varepsilon^{2}}-\frac{d \log g_{i}\left(\varepsilon_{i, t}\right)}{d \varepsilon}\right] \varepsilon_{t}^{\prime}\right\} C_{0}^{\prime} \hat{\delta}_{j}=0, i<j .
\end{aligned}
$$


Let us now introduce the effect of the number of observations. We get:

$$
\begin{aligned}
& \frac{1}{\sqrt{T}} \sum_{t=1}^{T}\left[\varepsilon_{j, t} \frac{d \log g_{i}\left(\varepsilon_{i, t}\right)}{d \varepsilon}-\varepsilon_{i, t} \frac{d \log g_{j}\left(\varepsilon_{j, t}\right)}{d \varepsilon}\right] \\
+ & E_{0}\left\{\left[\varepsilon_{j, t} \frac{d^{2} \log g_{i}\left(\varepsilon_{i, t}\right)}{d \varepsilon^{2}}-\frac{d \log g_{j}\left(\varepsilon_{j, t}\right)}{d \varepsilon}\right] \varepsilon_{t}^{\prime}\right\} C_{0}^{\prime} \sqrt{T} \hat{\delta}_{i} \\
- & E_{0}\left\{\left[\varepsilon_{i, t} \frac{d^{2} \log g_{j}\left(\varepsilon_{j, t}\right)}{d \varepsilon^{2}}-\frac{d \log g_{i}\left(\varepsilon_{i, t}\right)}{d \varepsilon}\right] \varepsilon_{t}^{\prime}\right\} C_{0}^{\prime} \sqrt{T} \hat{\delta}_{j}=o_{p}(1) .
\end{aligned}
$$

We have:

$$
\text { i) } \frac{1}{\sqrt{T}} \sum_{t=1}^{T}\left[\varepsilon_{j, t} \frac{d \log g_{i}\left(\varepsilon_{i, t}\right)}{d \varepsilon}-\varepsilon_{i, t} \frac{d \log g_{j}\left(\varepsilon_{j, t}\right)}{d \varepsilon}\right] \underset{d}{\rightarrow} Z_{i, j}, i<j,
$$

where the random vector obtained by stacking the $Z_{i, j}$ is Gaussian with zero-mean and $\operatorname{Cov}\left(Z_{i, j}, Z_{k, l}\right)=$ $\Omega_{(i, j),(k, l)}$, where

$$
\begin{aligned}
\Omega_{(i, j),(k, l)} & =0, \text { if } i<j, \quad k<l, \quad i, j, k, l \text { different, } \\
\Omega_{(i, j),(i, l)} & =E_{0}\left[\frac{d \log g_{j}\left(\varepsilon_{j, t}\right)}{d \varepsilon}\right] E_{0}\left[\frac{d \log g_{l}\left(\varepsilon_{l, t}\right)}{d \varepsilon}\right], \text { if } j \neq l, \\
\Omega_{(i, j),(k, j)} & =E_{0}\left[\frac{d \log g_{i}\left(\varepsilon_{i, t}\right)}{d \varepsilon}\right] E_{0}\left[\frac{d \log g_{k}\left(\varepsilon_{k, t}\right)}{d \varepsilon}\right], \text { if } i \neq k, \\
\Omega_{(i, j),(i, j)} & =E_{0}\left(\left[\frac{d \log g_{i}\left(\varepsilon_{i, t}\right)}{d \varepsilon}\right]^{2}\right)+E_{0}\left(\left[\frac{d \log g_{j}}{d \varepsilon}\left(\varepsilon_{j, t}\right)\right]^{2}\right), \\
& -2 E_{0}\left[\varepsilon_{i, t} \frac{d \log g_{i}\left(\varepsilon_{i, t}\right)}{d \varepsilon}\right] E_{0}\left[\varepsilon_{j, t} \frac{d \log g_{j}\left(\varepsilon_{j, t}\right)}{d \varepsilon}\right], \\
\Omega_{(i, j),(k, i)} & \left.=-E_{0}\left[\frac{d \log g_{j}\left(\varepsilon_{j, t}\right)}{d \varepsilon}\right] E_{0}\left[\frac{d \log g_{k}\left(\varepsilon_{k, t}\right)}{d \varepsilon}\right] \text { (with necessarily } k<j\right), \\
\Omega_{(i, j),(j, l)} & \left.=-E_{0}\left[\frac{d \log g_{i}\left(\varepsilon_{i, t}\right)}{d \varepsilon}\right] E_{0}\left[\frac{d \log g_{l}\left(\varepsilon_{l, t}\right)}{d \varepsilon}\right] \text { (with necessarily } i<l\right) .
\end{aligned}
$$


ii) Let us now denote:

$$
\begin{aligned}
& a_{i, j}^{\prime}=E_{0}\left\{-\left[\varepsilon_{j, t} \frac{d^{2} \log g_{i}\left(\varepsilon_{i, t}\right)}{d \varepsilon^{2}}-\frac{d \log g_{j}\left(\varepsilon_{j, t}\right)}{d \varepsilon}\right] \varepsilon_{t}^{\prime}\right\} C_{0}^{\prime} \\
& =\left\{E_{0}\left[-\frac{d^{2} \log g_{i}\left(\varepsilon_{i, t}\right)}{d \varepsilon^{2}}\right]+E_{0}\left[\varepsilon_{j, t} \frac{d \log g_{j}\left(\varepsilon_{j, t}\right)}{d \varepsilon}\right]\right\} c_{j, 0}^{\prime} .
\end{aligned}
$$

Then, $\forall i<j$,

$$
a_{i, j}^{\prime} \sqrt{T} \hat{\delta}_{i}-a_{j, i}^{\prime} \sqrt{T} \hat{\delta}_{j} \underset{d}{\rightarrow} Z_{i, j} .
$$

Let us introduce the notations:

$$
\begin{aligned}
& \hat{\delta}_{T}=\left(\hat{\delta}_{1}^{\prime}, \ldots, \hat{\delta}_{n}^{\prime}\right)^{\prime}, \delta \text { is a } n^{2} \text { dimensional vector, } \\
& Z=\left(Z_{1,2}, \ldots, Z_{1, n}, Z_{2,3}, \ldots, Z_{2, n}, \ldots, Z_{n-1, n}\right)^{\prime}
\end{aligned}
$$

where $Z$ is a $n(n-1) / 2$ dimensional vector,

$$
A_{1}=\left[\begin{array}{lllllll}
a_{1,2}^{\prime} & -a_{2,1}^{\prime} & 0 & \ldots & \ldots & 0 & 0 \\
a_{1,3}^{\prime} & 0 & -a_{3,1}^{\prime} & & & & \\
a_{1, n}^{\prime} & \ldots & \ldots & \ldots & \ldots & \ldots & -a_{n, 1}^{\prime} \\
0 & a_{2,3}^{\prime} & -a_{3,2}^{\prime} & \ldots & & 0 & 0 \\
0 & a_{2,4}^{\prime} & 0 & -a_{4,2}^{\prime} & \ldots & 0 & 0 \\
& \ldots & \ldots & \ldots & \ldots & \ldots & \\
0 & a_{2, n}^{\prime} & 0 & \ldots & \ldots & 0 & -a_{n, 2}^{\prime} \\
& \ldots & \ldots & \ldots & \ldots & \ldots & \\
0 & 0 & 0 & \ldots & \ldots & a_{n-1, n}^{\prime} & -a_{n, n-1}^{\prime}
\end{array}\right]
$$

where $A_{1}$ is $\left[n(n-1) / 2, n^{2}\right]$ matrix, 


$$
A_{2}=\left[\begin{array}{ccccccc}
c_{2,0}^{\prime} & c_{1,0}^{\prime} & 0 & \ldots & \ldots & 0 & 0 \\
c_{3,0}^{\prime} & 0 & c_{1,0}^{\prime} & \ldots & \ldots & 0 & 0 \\
& \ldots & \ldots & \ldots & \ldots & \ldots & \\
c_{n, 0}^{\prime} & \ldots & \ldots & \ldots & \ldots & 0 & c_{1,0}^{\prime} \\
0 & c_{3,0}^{\prime} & c_{2,0}^{\prime} & \ldots & \ldots & 0 & 0 \\
0 & c_{4,0}^{\prime} & 0 & c_{2,0}^{\prime} & \ldots & 0 & 0 \\
& \ldots & \ldots & \ldots & \ldots & \ldots & \\
0 & c_{n, 0}^{\prime} & 0 \ldots & \ldots & \ldots & 0 & c_{2,0}^{\prime} \\
& \ldots & \ldots & \ldots & \ldots & \ldots & \\
0 & 0 & 0 & 0 & & c_{n, 0}^{\prime} & c_{n-1,0}^{\prime}
\end{array}\right]
$$

where $A_{2}$ is a $\left[\frac{n(n-1)}{2}, n^{2}\right]$ matrix, and

$$
A_{3}=\left[\begin{array}{ccccc}
c_{1,0}^{\prime} & 0 & \ldots & \ldots & 0 \\
0 & c_{2,0}^{\prime} & \ldots & 0 & \\
\ldots & \ldots & \ldots & \ldots & \ldots \\
0 & \ldots & \ldots & \ldots & c_{n, 0}^{\prime}
\end{array}\right]
$$

where $A_{3}$ is a $\left(n, n^{2}\right)$ matrix.

Then we have:

$$
A \sqrt{T} \hat{\delta}_{T} \underset{d}{\rightarrow}\left(\begin{array}{c}
Z \\
0
\end{array}\right)
$$

where $A=\left(\begin{array}{c}A_{1} \\ A_{2} \\ A_{3}\end{array}\right)$ is a $\left(n^{2}, n^{2}\right)$ matrix or, equivalently,

$$
\sqrt{T} \hat{\delta}_{T} \rightarrow A^{-1}\left(\begin{array}{c}
Z \\
0
\end{array}\right)
$$


Noting that $\Omega=V(Z)$ is obtained from the terms $\Omega_{(i, j),(k, l)}$ given above, we get the asymptotic distribution of $\sqrt{T} \hat{\delta}_{T}$ :

$$
\sqrt{T} \hat{\delta}_{T} \approx N\left[0, A^{-1}\left(\begin{array}{cc}
\Omega & 0 \\
0 & 0
\end{array}\right) A^{\prime-1}\right]
$$

which is a Gaussian distribution on a vector subspace of dimension $n(n-1) / 2$.

As noted in Pham, Garat (1997), Section 2.B, the first-order expansion of the finite sample FOC depends on $\hat{\delta}_{i}=\hat{c}_{i}-c_{i, 0}$ by means of the quantities $c_{j, 0}^{\prime} \hat{\delta}_{i}=c_{j, 0}^{\prime}\left(\hat{c}_{i}-c_{i 0,}\right)$, which are simply the opposite of the elements in the first-order expansion of the contamination coefficients $\hat{\Delta}_{T}=I d-C_{0}^{-1} \hat{C}_{T}=I d-C_{0}^{\prime} \hat{C}_{T}$.

Since $\hat{C}_{T}=C_{0}+\left(\hat{\delta}_{1}, \ldots, \hat{\delta}_{n}\right)$, we have: $\hat{\Delta}_{i, j}=-c_{i, 0}^{\prime} \hat{\delta}_{j}$. We have the following results:

i) The asymptotic distribution of $\hat{\Delta}_{T}$ is degenerate, since

$$
\sqrt{T}\left(\hat{\Delta}_{i, j}+\hat{\Delta}_{j, i}\right)=o_{p}(1), i<j, \quad \text { and } \quad \sqrt{T}\left(\hat{\Delta}_{i, i}\right)=o_{p}(1), i=1, \ldots, n,
$$

due to the expansion of the conditions for the orthogonal matrix $\hat{C}_{T}$.

ii) Thus, the asymptotic distribution of $\hat{\Delta}_{T}$ is known whenever we know the asymptotic distribution of its strictly lower triangular part, that is, of the $\hat{\Delta}_{i, j}, i<j$.

iii) The joint distribution of the $\hat{\Delta}_{i, j}, i<j$, is easily deduced by using the definition of $a_{i, j}$ and the convergence:

$$
a_{i, j}^{\prime} \sqrt{T} \hat{\delta}_{i}-a_{j, i}^{\prime} \sqrt{T} \hat{\delta}_{j} \underset{d}{\rightarrow} Z_{i, j}
$$

We get:

$$
\sqrt{T} E_{0}\left[\frac{d^{2} \log g_{i}\left(\varepsilon_{i, t}\right)}{d \varepsilon^{2}}+\frac{d^{2} \log g_{j}\left(\varepsilon_{j, t}\right)}{d \varepsilon^{2}}-\varepsilon_{j, t} \frac{d \log g_{j}\left(\varepsilon_{j, t}\right)}{d \varepsilon}-\varepsilon_{i, t} \frac{d \log g_{i}\left(\varepsilon_{i, t}\right)}{d \varepsilon}\right] \hat{\Delta}_{i, j} \underset{d}{\rightarrow} Z_{i, j} .
$$

The factor multiplying $\hat{\Delta}_{i, j}$ is nonzero, because of the local concavity condition, and the asymptotic distribution of the $\hat{\Delta}_{i, j}, i<j$, is derived.

As in Pham, Garat (1997), the asymptotic distribution of the $\hat{\Delta}_{i, j}$ no longer depends on matrix $C_{0}$, but just on the distributional properties of the sources and on the choice of the pseudo p.d.f..

Our results have taken explicitly into account the constraints of orthogonal matrix $C$ in the first-order conditions. In this respect our expansions differ from the expansions in Pham, Garat (1997) or Wei (2014) as well as the associated asymptotic distribution of the estimators. 


\section{Appendix 5}

\section{Asymptotic Variance of the PML Estimator for $n=2$.}

\section{A.5.1. Derivation of the asymptotic variance}

When $n=2$, the orthogonal matrix $C$ (with $\operatorname{det} C=1)$ can be parametrized as:

$$
C(\theta)=\left(\begin{array}{cc}
\cos \theta & -\sin \theta \\
\sin \theta & \cos \theta
\end{array}\right)
$$

and the pseudo log-likelihood function written as:

$$
L_{T}(\boldsymbol{\theta})=\sum_{t=1}^{T}\left\{\log g_{1}\left[c_{1}^{\prime}(\boldsymbol{\theta}) y_{t}\right]+\log g_{2}\left[c_{2}^{\prime}(\boldsymbol{\theta}) y_{t}\right]\right\} \equiv \sum_{t=1}^{T} \log f\left(y_{t} ; \boldsymbol{\theta}\right) .
$$

The PML estimator of parameter $\theta$ is asymptotically normal with variance:

$$
V_{a s}\left[\sqrt{T}\left(\hat{\theta}_{T}-\theta_{0}\right)\right]=J^{-2} I,
$$

where

$$
J=E_{0}\left[\frac{-\partial^{2} \log f\left(Y_{t} ; \theta_{0}\right)}{\partial \theta^{2}}\right], \quad I=E_{0}\left(\left[\frac{\partial \log f\left(Y_{t} ; \theta_{0}\right)}{\partial \theta}\right]^{2}\right)
$$

We have:

$$
\begin{aligned}
\frac{\partial \log f\left(y_{t} ; \theta\right)}{\partial \theta} & =\sum_{i=1}^{2}\left\{\frac{d \log g_{i}}{d \varepsilon}\left[c_{i}^{\prime}(\theta) y_{t}\right] \frac{d c_{i}^{\prime}(\theta)}{d \theta} y_{t}\right\} \\
\frac{\partial^{2} \log f\left(y_{t} ; \theta\right)}{\partial \theta^{2}} & =\sum_{i=1}^{2}\left\{\frac{d \log g_{i}}{d \varepsilon}\left[c_{i}^{\prime}(\theta) y_{t}\right] \frac{d^{2} c_{i}^{\prime}(\theta)}{d \theta^{2}} y_{t}\right. \\
& \left.+\frac{d^{2} \log g_{i}}{d \varepsilon^{2}}\left[c_{i}^{\prime}(\theta) y_{t}\right]\left[\frac{d c_{i}^{\prime}(\theta)}{d \theta} y_{t}\right]^{2}\right\} .
\end{aligned}
$$

It is easily checked that:

$$
\frac{d C^{\prime}(\theta)}{d \theta} C(\theta)=\left(\begin{array}{cc}
0 & 1 \\
-1 & 0
\end{array}\right), \frac{d^{2} C^{\prime}(\theta)}{d \theta^{2}} C(\theta)=-I d .
$$


We deduce that:

$$
\begin{aligned}
\frac{\partial \log f\left(y_{t} ; \theta_{0}\right)}{\partial \theta} & =\frac{d \log g_{1}\left(\varepsilon_{1, t}\right)}{d \varepsilon} \varepsilon_{2, t}-\frac{d \log g_{2}\left(\varepsilon_{2, t}\right)}{d \varepsilon} \varepsilon_{1, t} \\
\frac{\partial^{2} \log f\left(y_{t} ; \theta_{0}\right)}{\partial \theta^{2}} & =-\frac{d \log g_{1}\left(\varepsilon_{1, t}\right)}{d \varepsilon} \varepsilon_{1, t}-\frac{d \log g_{2}\left(\varepsilon_{2, t}\right)}{d \varepsilon} \varepsilon_{2, t} \\
& +\frac{d^{2} \log g_{1}\left(\varepsilon_{1, t}\right)}{d \varepsilon^{2}} \varepsilon_{2, t}^{2}+\frac{d^{2} \log g_{2}\left(\varepsilon_{2, t}\right)}{d \varepsilon^{2}} \varepsilon_{1, t}^{2} .
\end{aligned}
$$

Thus:

$$
\begin{aligned}
I= & E_{0}\left[\left(\frac{d \log g_{1}\left(\varepsilon_{1, t}\right)}{d \varepsilon}\right)^{2}\right]+E_{0}\left[\left(\frac{d \log g_{2}\left(\varepsilon_{2, t}\right)}{d \varepsilon}\right)^{2}\right] \\
& -2 E_{0}\left[\varepsilon_{1, t} \frac{d \log g_{1}\left(\varepsilon_{1, t}\right)}{d \varepsilon}\right] E_{0}\left[\varepsilon_{2, t} \frac{d \log g_{2}\left(\varepsilon_{2, t}\right)}{d \varepsilon}\right] \\
J= & E_{0}\left[\varepsilon_{1, t} \frac{d \log g_{1}\left(\varepsilon_{1, t}\right)}{d \varepsilon}\right]+E_{0}\left[\varepsilon_{2, t} \frac{d \log g_{2}\left(\varepsilon_{2, t}\right)}{d \varepsilon}\right] \\
& -E_{0}\left[\frac{d^{2} \log g_{1}\left(\varepsilon_{1, t}\right)}{d \varepsilon^{2}}\right]-E_{0}\left[\frac{d^{2} \log g_{2}\left(\varepsilon_{2, t}\right)}{d \varepsilon^{2}}\right] .
\end{aligned}
$$

The asymptotic distribution of $\hat{C}_{T}=C\left(\hat{\theta}_{T}\right)$ is deduced by the $\delta$-method, noting that:

$$
\frac{d C(\theta)}{d \theta}=\left(\begin{array}{cc}
-\sin \theta & -\cos \theta \\
\cos \theta & -\sin \theta
\end{array}\right)=\left[c_{2}(\theta),-c_{1}(\theta)\right]
$$

We get:

$$
\begin{aligned}
& V_{a s}\left[\sqrt{T}\left(\operatorname{vec} \hat{C}_{T}-\operatorname{vec} C_{0}\right)\right] \\
= & I / J^{2} \operatorname{vec}\left(\frac{d C\left(\theta_{0}\right)}{d \theta}\right) \operatorname{vec}\left(\frac{d C\left(\theta_{0}\right)}{d \theta}\right)^{\prime} . \\
= & I / J^{2}\left[\begin{array}{cc}
c_{2}\left(\theta_{0}\right) c_{2}^{\prime}\left(\theta_{0}\right) & -c_{2}\left(\theta_{0}\right) c_{1}^{\prime}\left(\theta_{0}\right) \\
-c_{1}\left(\theta_{0}\right) c_{2}^{\prime}\left(\theta_{0}\right) & c_{1}\left(\theta_{0}\right) c_{1}^{\prime}\left(\theta_{0}\right)
\end{array}\right] .
\end{aligned}
$$

Let us finally discuss the expressions of $I$ and $J$, when $g_{i}=f_{i, 0}$ is the true distribution. We can construct different parametric models from distribution $f_{0}$, that are:

- a model with drift parameter $f_{0}(\varepsilon-m)$;

- a model with scale parameter $c f_{0}(c \varepsilon)$. 
From the model with drift parameter, we deduce:

$$
E_{m}\left[\left(\frac{\partial \log f_{0}(\varepsilon-m)}{\partial m}\right)^{2}\right]=E\left[-\frac{\partial^{2} \log f_{0}(\varepsilon-m)}{\partial m^{2}}\right],
$$

which, for $m=0$, implies:

$$
E_{0}\left[\frac{d \log f_{0}(\varepsilon)}{d \varepsilon}\right]^{2}=E_{0}\left[\frac{-d^{2} \log f_{0}(\varepsilon)}{d \varepsilon^{2}}\right] .
$$

From the model with scale parameter, we deduce a zero-mean score:

$$
E_{c}\left[\frac{1}{c}+\varepsilon \frac{d \log f_{0}(c \varepsilon)}{d \varepsilon}\right]=0
$$

which implies, for $c=1$ :

$$
E_{0}\left[\varepsilon \frac{d \log f_{0}(\varepsilon)}{d \varepsilon}\right]=-1
$$

Thus, if $g_{i}=f_{i, 0}, i=1,2$, we get as expected the same value for $I$ and $J$ :

$$
I=J=\sum_{i=1}^{2} E_{0}\left[-\frac{d^{2} \log f_{i, 0}\left(\varepsilon_{i, t}\right)}{d \varepsilon^{2}}-1\right] \text {. }
$$

\section{A.5.2. Asymptotic variance of the contamination coefficients.}

Let us denote by $c_{0}^{1}, c_{0}^{2}$ the rows of matrix $C_{0}^{-1}$. We have:

$$
c_{0}^{j} c_{0, j}=1, \text { for } j=1,2 \text {, and } c_{0}^{j} c_{0, i}=0 \text {, for } i \neq j .
$$

With these notations, we get: $C_{0}^{-1} \hat{C}_{T}=\left(\begin{array}{c}c_{0}^{1} \\ c_{0}^{2}\end{array}\right)\left(\hat{c}_{1}, \hat{c}_{2}\right)=\left(\begin{array}{cc}c_{0}^{1} \hat{c}_{1} & c_{0}^{1} \hat{c}_{2} \\ c_{0}^{2} \hat{c}_{1} & c_{0}^{2} \hat{c}_{2}\end{array}\right)$, and vec $\left(C_{0}^{-1} \hat{C}_{T}\right)=$ $\left(c_{0}^{1} \hat{c}_{1}, c_{0}^{2} \hat{c}_{1}, c_{0}^{1} \hat{c}_{2}, c_{0}^{2} \hat{c}_{2}\right)^{\prime}$.

The elements of the asymptotic variance $v e c \hat{\Delta}_{T}$ are equal to the elements of the asymptotic variance of $\operatorname{vec}\left(C_{0}^{-1} \hat{C}_{T}\right)$. They are easily computed. For instance we have:

$$
\begin{aligned}
V_{a s}\left[\sqrt{T}\left(c_{0}^{1} \hat{c}_{1}-1\right)\right] & =\frac{\omega^{2}}{\left(\gamma_{1,2}+\gamma_{2,1}\right)^{2}} c_{0}^{1} c_{0,2} c_{0,2}^{\prime}\left(c_{0}^{1}\right)^{\prime}=0 \\
V_{a s}\left[\sqrt{T} c_{0}^{2} \hat{c}_{1}\right] & =\frac{\omega^{2}}{\left(\gamma_{1,2}+\gamma_{2,1}\right)^{2}} c_{0}^{2} c_{0,2} c_{0,2}^{\prime}\left(c_{0}^{2}\right)^{\prime}=\frac{\omega^{2}}{\left(\gamma_{1,2}+\gamma_{2,1}\right)^{2}},
\end{aligned}
$$

and so on. 


\section{Appendix 6}

\section{Expansion of the Empirical Covariance}

Let us consider i.i.d. observations $\left(X_{t}, Y_{t}\right), t=1, \ldots, T$. Their empirical covariance can be expanded for large $T$ as:

$$
\begin{aligned}
& \sqrt{T}[\widehat{\operatorname{Cov}}(X, Y)-\operatorname{Cov}(X, Y)] \\
= & \sqrt{T}\left\{\frac{1}{T} \sum_{t=1}^{T}\left[X_{t} Y_{t}-E(X Y)\right]-\frac{1}{T} \sum_{t=1}^{T} X_{t} \frac{1}{T} \sum_{t=1}^{T} Y_{t}+E X E Y\right\} \\
\simeq & \sqrt{T}\left\{\frac{1}{T} \sum_{t=1}^{T}\left[X_{t} Y_{t}-E(X Y)\right]-\frac{1}{T} \sum_{t=1}^{T}\left(X_{t}-E X\right) E Y-\frac{1}{T} \sum_{t=1}^{T}\left(Y_{t}-E Y\right) E X\right\}+o_{P}(1) \\
= & \frac{1}{\sqrt{T}} \sum_{t=1}^{T}\left[\left(X_{t}-E X\right)\left(Y_{t}-E Y\right)-\operatorname{Cov}(X, Y)\right]+o_{P}(1) .
\end{aligned}
$$

This expansion can be used to compute the asymptotic variance of an empirical covariance as well as the asymptotic covariance between two empirical covariances. For instance we have:

$$
\begin{aligned}
& V_{a s}[\sqrt{T}[\widehat{\operatorname{Cov}}(X, Y)-\operatorname{Cov}(X, Y)]]=V[(X-E X)(Y-E Y)], \\
& \operatorname{Cov}_{a s}\{\sqrt{T}[\widehat{\operatorname{Cov}}(X, Y)-\operatorname{Cov}(X, Y) \mid, \sqrt{T}[\widehat{\operatorname{Cov}}(Z, U)-\operatorname{Cov}(Z, U)]\} \\
& =\operatorname{Cov}[(X-E Y)(Y-E Y),(Z-E Z)(U-E U)]
\end{aligned}
$$




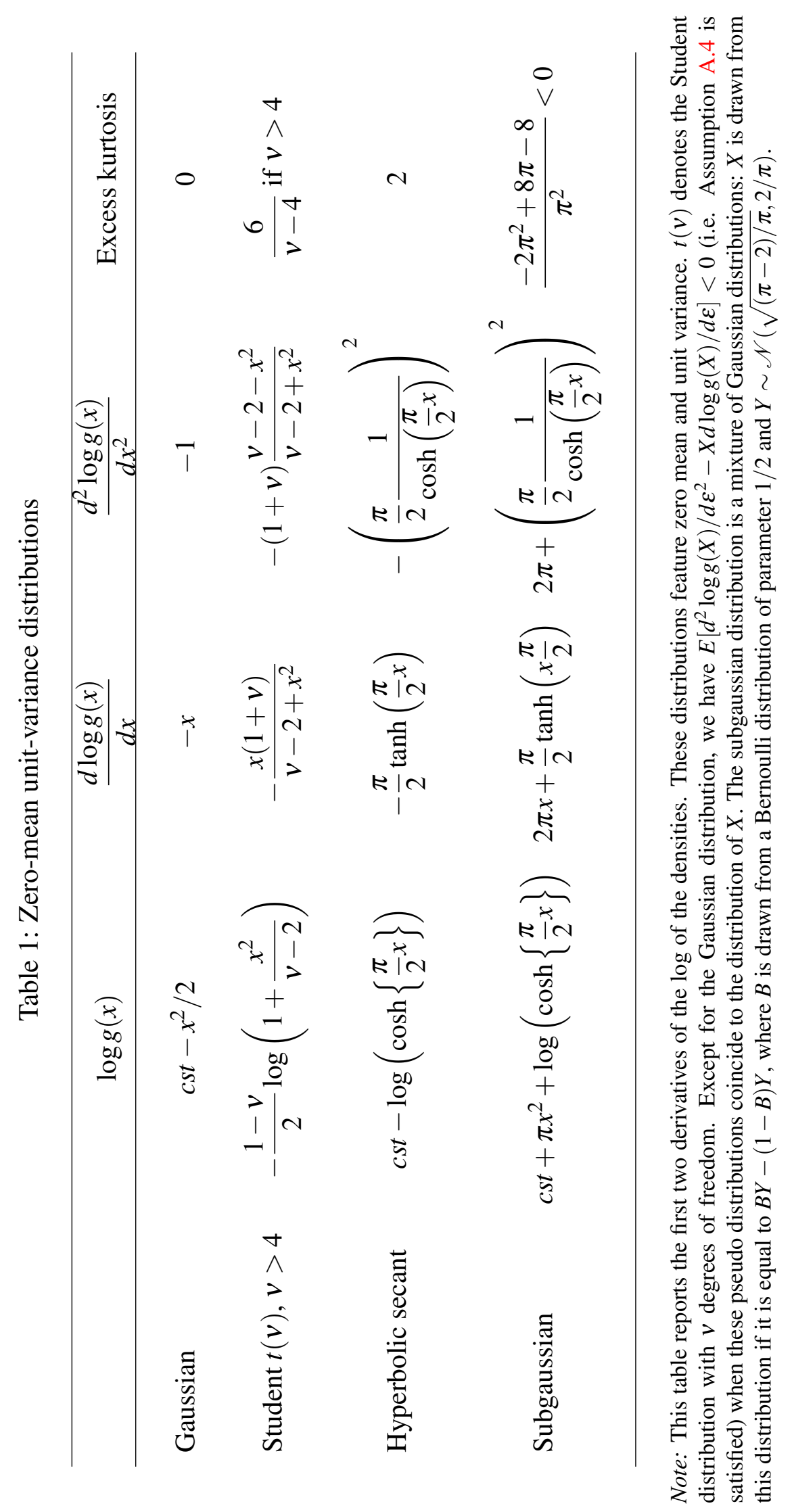




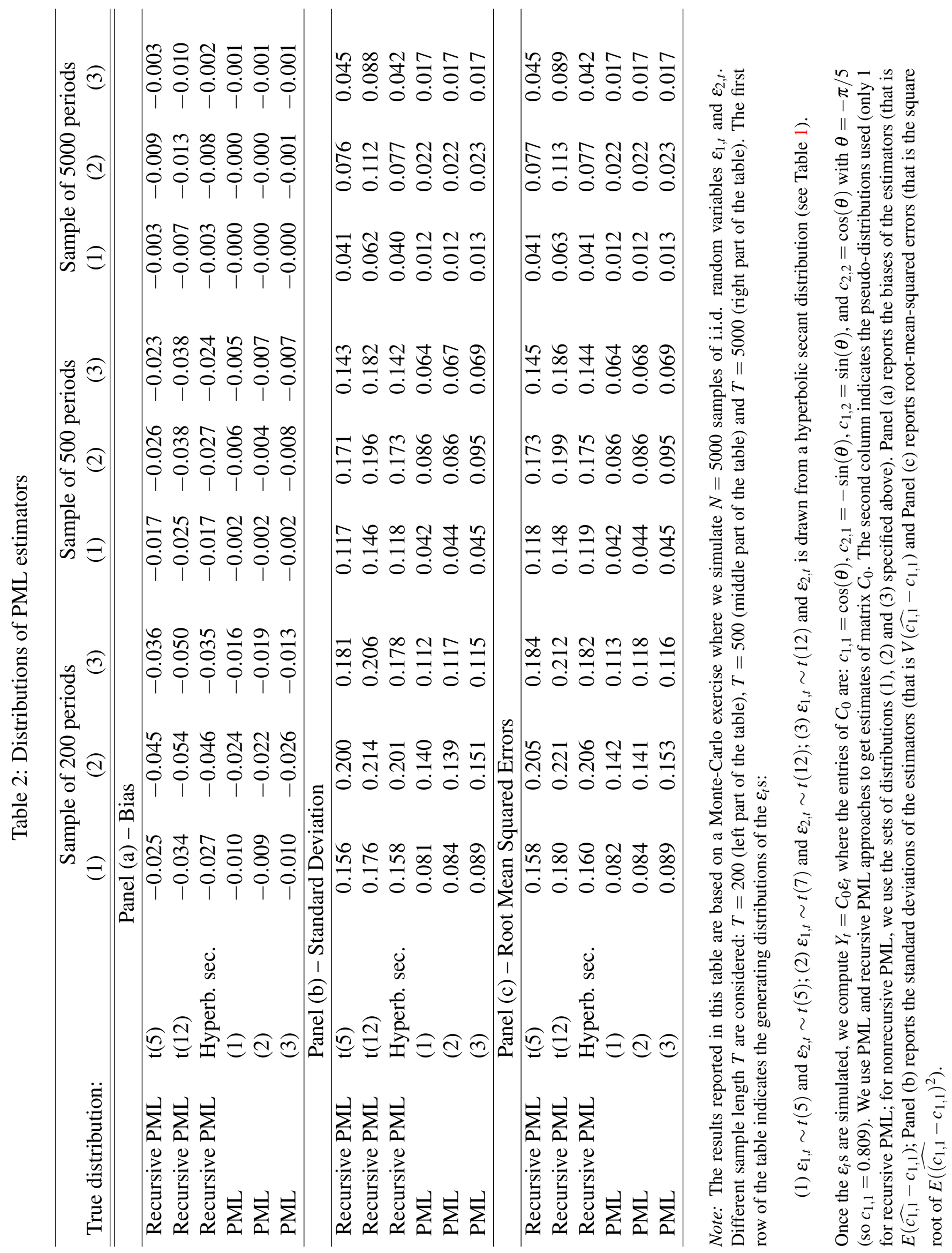


Table 3: Asymptotic versus finite-sample PML estimator distributions

\begin{tabular}{|c|c|c|c|c|c|c|c|c|c|}
\hline \multirow{2}{*}{$\begin{array}{l}\text { True distribution: } \\
\text { Pseudo distribution: }\end{array}$} & \multicolumn{3}{|c|}{ (1) } & \multicolumn{3}{|c|}{ (2) } & \multicolumn{3}{|c|}{ (3) } \\
\hline & (1) & (2) & (3) & (1) & (2) & (3) & (1) & (2) & (3) \\
\hline & \multicolumn{9}{|c|}{ Samples of 200 periods } \\
\hline$\alpha=25 \%$ & 0.26 & 0.24 & 0.26 & 0.30 & 0.25 & 0.32 & 0.30 & 0.31 & 0.24 \\
\hline$\alpha=50 \%$ & 0.49 & 0.47 & 0.48 & 0.50 & 0.45 & 0.51 & 0.52 & 0.53 & 0.43 \\
\hline$\alpha=75 \%$ & 0.71 & 0.69 & 0.69 & 0.68 & 0.63 & 0.67 & 0.71 & 0.70 & 0.63 \\
\hline$\alpha=90 \%$ & 0.83 & 0.81 & 0.81 & 0.78 & 0.74 & 0.77 & 0.83 & 0.82 & 0.76 \\
\hline \multirow[t]{2}{*}{$\alpha=95 \%$} & 0.88 & 0.86 & 0.86 & 0.83 & 0.80 & 0.81 & 0.87 & 0.86 & 0.83 \\
\hline & \multicolumn{9}{|c|}{ Samples of 500 periods } \\
\hline$\alpha=25 \%$ & 0.25 & 0.25 & 0.26 & 0.29 & 0.25 & 0.34 & 0.27 & 0.31 & 0.23 \\
\hline$\alpha=50 \%$ & 0.50 & 0.48 & 0.50 & 0.51 & 0.47 & 0.57 & 0.51 & 0.56 & 0.44 \\
\hline$\alpha=75 \%$ & 0.73 & 0.72 & 0.73 & 0.72 & 0.68 & 0.75 & 0.74 & 0.77 & 0.67 \\
\hline$\alpha=90 \%$ & 0.87 & 0.86 & 0.86 & 0.83 & 0.81 & 0.84 & 0.86 & 0.88 & 0.82 \\
\hline \multirow[t]{2}{*}{$\alpha=95 \%$} & 0.92 & 0.91 & 0.91 & 0.88 & 0.86 & 0.88 & 0.91 & 0.92 & 0.88 \\
\hline & \multicolumn{9}{|c|}{ Samples of 5000 periods } \\
\hline$\alpha=25 \%$ & 0.25 & 0.25 & 0.25 & 0.26 & 0.24 & 0.30 & 0.26 & 0.28 & 0.24 \\
\hline$\alpha=50 \%$ & 0.50 & 0.50 & 0.50 & 0.50 & 0.47 & 0.57 & 0.50 & 0.56 & 0.49 \\
\hline$\alpha=75 \%$ & 0.74 & 0.74 & 0.74 & 0.75 & 0.72 & 0.81 & 0.75 & 0.82 & 0.74 \\
\hline$\alpha=90 \%$ & 0.89 & 0.89 & 0.89 & 0.89 & 0.87 & 0.93 & 0.90 & 0.94 & 0.88 \\
\hline$\alpha=95 \%$ & 0.95 & 0.94 & 0.94 & 0.94 & 0.93 & 0.97 & 0.95 & 0.98 & 0.94 \\
\hline
\end{tabular}

Note: This table assesses the finite-sample adequacy of the asymptotic distribution of the PML estimators. It is based on the same Monte-Carlo exercise as the one detailed in Table 2. The first two rows of the table respectively indicate which set of distributions is used to draw the $\varepsilon_{t}$ s and which one is used for the pseudo-distributions. These sets of distributions [denoted by (1), (2) and (3)] are as follows:

(1) $\varepsilon_{1, t} \sim t(5)$ and $\varepsilon_{2, t} \sim t(5)$;

(2) $\varepsilon_{1, t} \sim t(7)$ and $\varepsilon_{2, t} \sim t(12)$;

(3) $\varepsilon_{1, t} \sim t(12)$ and $\varepsilon_{2, t}$ is drawn from an hyperbolic secant distribution.

For each simulated sample, (a) we compute the PML estimates of matrix $C_{0}$ and (b) we use the formulas given in Appendix 4 to compute ${\widehat{\sigma_{1,1}}}^{2}$, the asymptotic variance of $\widehat{c_{1,1}}$ (the PML estimate of $c_{1,1}$ ), and (c) we look whether the true value of $c_{1,1}$ lies in the interval $\left[\widehat{c_{1,1}}-\phi_{\alpha / 2} \widehat{\sigma_{1,1}}, \widehat{c_{1,1}}+\phi_{\alpha / 2} \widehat{\sigma_{1,1}}\right]$, where $\phi_{\alpha}$ is the $\alpha^{\text {th }}$ quantile of the standard normal distribution. The values of $\alpha$ are given in the first column of the table. The figures reported in the table correspond to the fractions of simulations for which $c_{1,1}$ lies in the interval. If the distribution of the finite-sample estimates of $c_{1,1}$ were equal to the asymptotic one, the figures reported in the table would be equal to $\alpha$. 


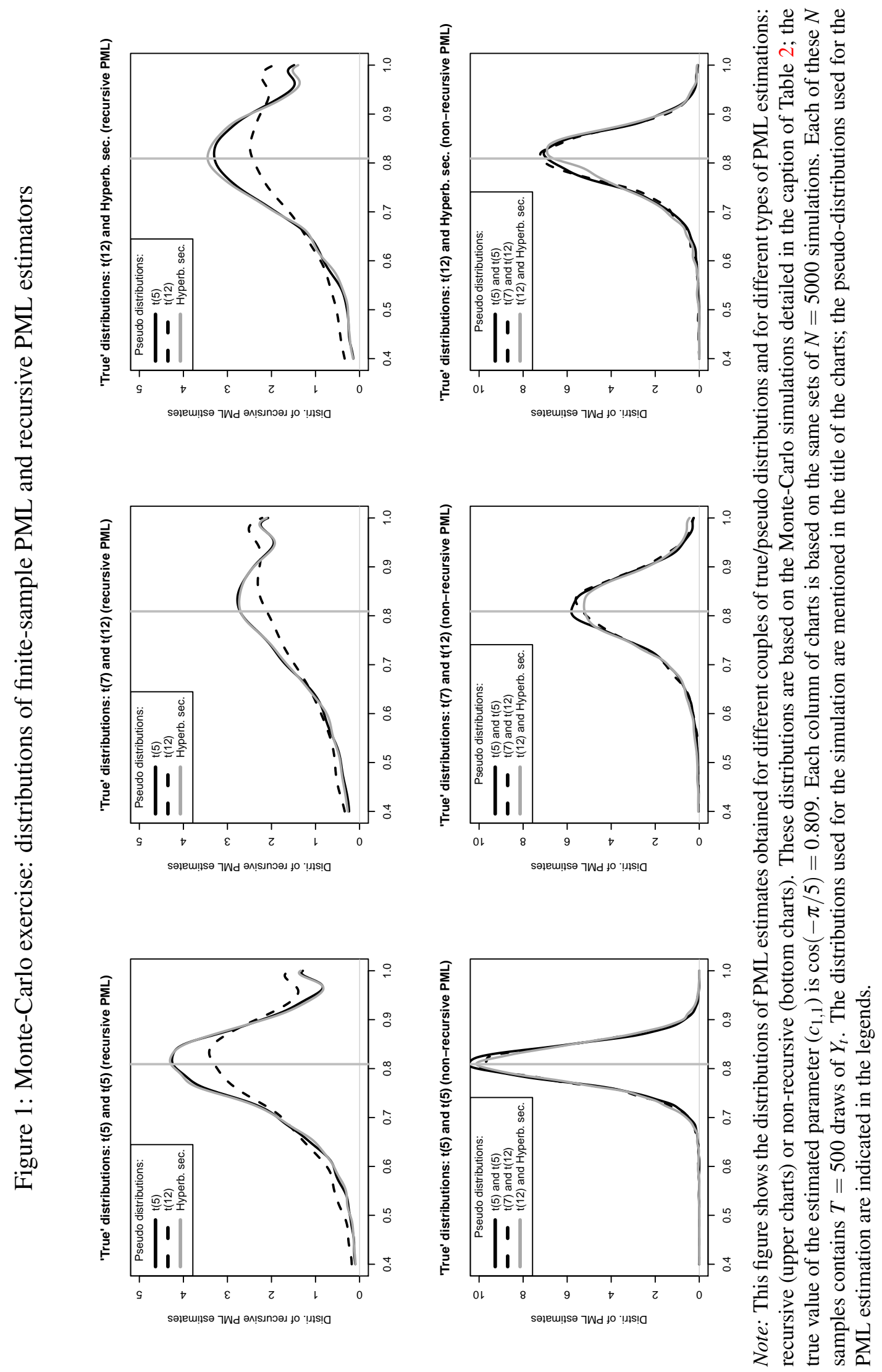


Table 4: Independent Component Analysis of VAR residuals

\begin{tabular}{lrrrr}
\hline \multicolumn{3}{c}{$\begin{array}{c}\text { Real activity measured by: } \\
\text { Output gap }\end{array}$} & \multicolumn{2}{c}{$\begin{array}{c}\text { Real activity measured by: } \\
\text { Unempl. gap }\end{array}$} \\
\hline & Parameter & Stand. dev. & Parameter & Stand. dev. \\
\hline & & & & \\
$c_{1,1}$ & 0.945 & 0.041 & 0.958 & 0.068 \\
$c_{2,1}$ & -0.324 & 0.121 & 0.249 & 0.248 \\
$c_{3,1}$ & 0.036 & 0.076 & 0.145 & 0.094 \\
$c_{1,2}$ & 0.317 & 0.122 & -0.263 & 0.247 \\
$c_{2,2}$ & 0.940 & 0.042 & 0.961 & 0.065 \\
$c_{3,2}$ & 0.127 & 0.061 & 0.087 & 0.073 \\
$c_{1,3}$ & -0.075 & 0.073 & -0.117 & 0.090 \\
$c_{2,3}$ & -0.108 & 0.063 & -0.122 & 0.065 \\
$c_{3,3}$ & 0.991 & 0.008 & 0.986 & 0.015 \\
\hline
\end{tabular}

\begin{tabular}{lrr} 
Wald test $C=I d$ & {$[$ Stat. $(\mathrm{p}$-value $)]$} & [Stat. (p-value) $]$ \\
\hline Ordering: $Y_{t}=\left[\pi_{t}, y_{t}, r_{t}\right]$ & $10.64(0.014)$ & $5.35(0.148)$ \\
Ordering: $Y_{t}=\left[y_{t}, \pi_{t}, r_{t}\right]$ & $9.06(0.029)$ & $4.58(0.205)$ \\
\hline
\end{tabular}

Note: This table reports the results of ICA conducted on the residuals of vector autoregressive models. The three dependent variables of the 6-lag VARs are: inflation $\left(\pi_{t}\right)$, economic activity $\left(y_{t}\right)$ and a short-term rate $\left(r_{t}\right)$. Two proxies of economic activity are considered: the output gap (log difference between real GDP and potential GDP) and the unemployment gap (difference between the unemployment rate and the natural rate of unemployment). The three dependent variables are stacked in vector $Y_{t}=\left[\pi_{t}, y_{t}, r_{t}\right]$. Change in the oil price is added as an exogenous variable. We aim at estimating the orthogonal matrix $C$ that is such that the vector of residuals of the VAR model is given by $S C \varepsilon_{t}$ where $V\left(Y_{t} \mid X_{t}\right)=\Sigma=S S^{\prime}$ ( $X_{t}$ contains lagged values of the dependent variables and the contemporaneous change in oil price) and where $\left(\varepsilon_{t}\right)$ satisfies Assumption A.1. Standardized residuals are obtained by multiplying the (OLS-based) VAR residuals by $\hat{S}_{T}^{-1}$, where $\hat{S}_{T}$ is the lower triangular matrix resulting from the Cholesky decomposition of $\hat{\Sigma}_{T}$ (the empirical covariance matrix of the OLS residuals). Matrix $C$ is estimated by applying the PML approach on the series of standardized residuals. The pseudo density functions $g_{i}$ are those of three distinct and asymmetric mixtures of Gaussian distributions (see Footnote 21). Asymptotic standard deviations of the elements of $\hat{C}_{T}$ are obtained by using the formulas derived in Appendix 4 . The bottom of the table shows the results of tests where, under the null hypothesis, the ICA approach results in the same structural shocks as those stemming from shock identification schemes based on short-run restrictions (see Section 2.5 and Section 4.2 for details about these tests); $p$-values of the tests are given in parentheses. 


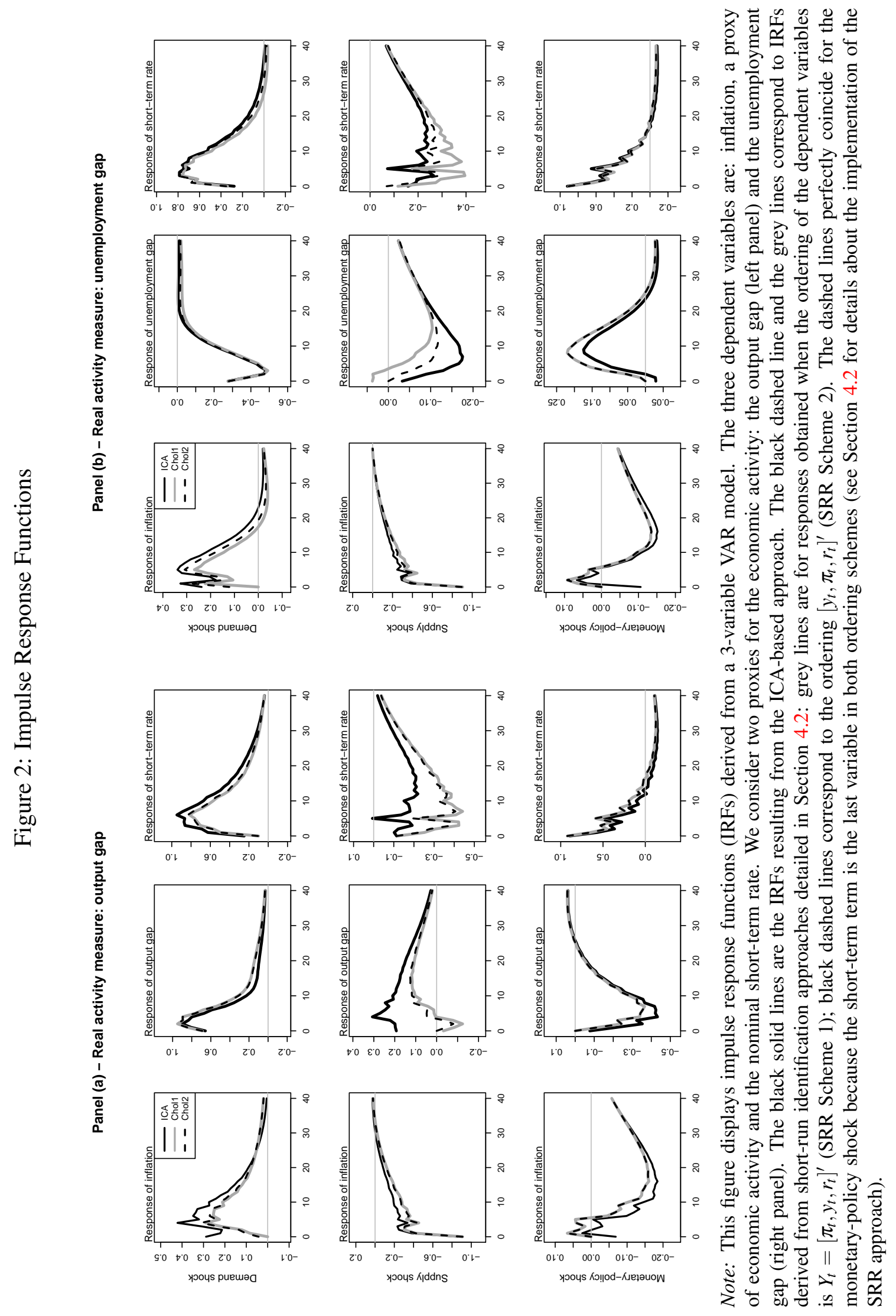

